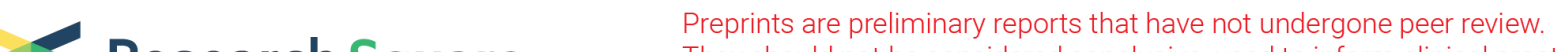 Research Square They should not be considered conclusive, used to inform clinical practice, or referenced by the media as validated information.
}

\section{Blocking PSD-93-CX3CL1 Interaction Promotes Phenotypic Polarization Transformation of Microglia During Acute Ischemic Stroke Injury}

Qingxiu Zhang ( $\square$ zhangqingxiu@163.com )

Nanjing University Medical School Affiliated Nanjing Drum Tower Hospital https://orcid.org/00000001-5285-2908

\section{Xiaowei Cao}

Nanjing University Medical School Affiliated Nanjing Drum Tower Hospital

Hui Yang

Nanjing University Medical School Affiliated Nanjing Drum Tower Hospital

Xiaomei Liu

Xuzhou Medical University

\section{Shiying Lou}

Nanjing University Medical School Affiliated Nanjing Drum Tower Hospital

Liping Kong

Xuzhou Medical University

\section{Liangqun Rong}

Xuzhou Medical University

Junjun Shan

Xuzhou Medical University

\section{Yun Xu}

Nanjing University Medical School Affiliated Nanjing Drum Tower Hospital

\section{Research}

Keywords: PSD-93, CX3CL1, Tat-CX3CL1 (357-395aa), cerebral ischemia-reperfusion, Microglia

Posted Date: May 25th, 2021

DOI: https://doi.org/10.21203/rs.3.rs-538353/v1

License: (c) (1) This work is licensed under a Creative Commons Attribution 4.0 International License.

Read Full License 


\section{Abstract}

Background囚Postsynaptic density 93 (PSD-93) plays an important role in ischemic brain injury through mediating neurotoxicity and neuroinflammation. Blocked the combination of PSD-93 and Fractalkine (CX3Cchemokineligand1, CX3CL1) play beneficial roles in acute ischemic stroke. However, the underlying mechanism still need further exploration.

Methods $\$ In this study, male C57BL/6 mice aged 8-12 weeks and weighted 22-26g were applied with Middle Cerebral Artery Occlusion (MCAO) model and randomly divided into different groups. Firstly, realtime quantitative PCR was to detect some cytokines associated with M1 and M2 type of microglia at different time points in transient MCAO model. Secondly, triphenyl tetrazolium chloride (TTC) staining, brain water content and behavioral assessments were used to evaluate the neurologic damage. Immunofluorescence staining was performed to measure the white matter injury, and microglia polarization. Moreover, enzyme-linked immunosorbent assay (ELISA) was used to investigate the expression of soluble CX3CL1.

Results $\triangle \mathrm{M} 1$ phenotype cytokines were detected at different time points and found that their expression increased from beginning at 6 hours after reperfusion and most peaked at $24 \mathrm{~h}$ after reperfusion. However, the expression of M2 phenotype cytokines decreased or still keep invariant at 6 hours and $24 \mathrm{~h}$ after reperfusion. Interestingly, Tat-CX3CL1 (357-395aa) can facilitate microglia polarization from M1 type to M2 type through inhibiting expression of the soluble CX3CL1. Furthermore, CX3CL1 specifiC cleavase inhibitor ADAM17 could restrain the microglia polarization transformation via reducing the soluble CX3CL1 formation. Moreover, Tat-CX3CL1 (357-395aa) also attenuate long-term cognitive deficits and improved white matter integrity.

Conclusions: In conclusion, we demonstrated that specific blockage the binding of PSD-93 and CX3CL1 by Tat-CX3CL1 (357-395aa) could perform functional recovery after ischemic stroke by promoting type microglia polarization transformation from M1 to M2. And the peptide Tat-CX3CL1 (357-395aa) is a promising strategy for treatment of ischemic stroke.

\section{Background}

Ischemic stroke, a leading cause of death and disability, induces neurotoxicity and neuroinflammation[14]. Inflammatory responses induced by microglia polarization exacerbate the cerebral infarction[5]. Microglial heterogeneity is of paramount importance for ischemic brain injury. And in ischemic stroke model, polarization of microglia toward M2 phenotype has been proved to be protective[6]. However, the polarization of microglia occurs dynamic changes and is highly dependent on environmental signals during cerebral ischemia-reperfusion[7, 8]. Therefore, intervention to promote the microglia to maintain their homeostatic phenotype may be a promising strategy to achieve successful functional recovery after stroke. 
Traditionally, microglia are considered to be specific tissue-resident macrophages in the central nervous system (CNS) and to quickly respond to pathological event $[8,9]$. In response to ischemic stroke, microglial cells initiate the production of pro-inflammatory mediators such as interleukin (IL)-1 $\beta$, tumor necrosis factor (TNF)- $a$, and IL-6, which means the M1 phenotype polarization[10, 11]. Contrastingly, M2 phenotype performs an anti-inflammatory role through producing cytokines including IL-4, IL-10, and transforming growth factor- $\beta$ (TGF- $\beta$ ) $[10,11]$. In recent years, considerable research efforts to elucidate the functions of microglia phenotypic polarization shift in ischemic brain injury has been devoted $[5,6$, 12-14]. However, the potential mechanism still needs further elusive.

Our previous study showed that PSD-93, acting as a PSDs scaffold protein, binds directly to 670-685 amino acid sequence of GTPase-activating protein for Ras (SynGAP) and promotes SynGAP ubiquitination in ischemic brain injury [15]. And knockout PSD-93 improves in neurological deficit by inhibiting pro-inflammatory factors and promotes the expression of anti-inflammatory factors[16]. Furthermore, we also found that PSD-93 interacts with CX3CL1 to mediate neuron-microglia crosstalk and initiate neuroinflammation[17]. Using yeast two hybrid, and co-immunoprecipitation assay, we confirmed the binding sites are located at the 420-535 amino acid sequence of PSD-93 and 357-395 amino acid sequence of CX3CL1[17]. Based on the above results, we constructed a small peptides TatCX3CL1 (357-395aa) to disturb the combination of PSD-93 and CX3CL1 and found that it could attenuate cerebral infarct volume. But the underlying protective mechanism need to further explore.

In the current study, we investigated the peptide Tat-CX3CL1 (357-395aa) perfects functional recovery after ischemic stroke by promoting M2 type microglia polarization. Delivery of Tat-CX3CL1 (357-395aa) inhibited the production of M1 type proinflammatory mediators, facilitated M2 type the anti-inflammatory cytokines and improved the integrity of the blood-brain barrier (BBB) after stroke. Furthermore, TatCX3CL1 (357-395aa) reduced the cerebral infarct volume and improved long-term cognitive function after stroke. Overall, these findings indicate that specific blockage of the binding of PSD-93 and CX3CL1 by Tat-CX3CL1 (357-395aa) may be a potential novel neurorestorative strategy for treatment of ischemic stroke.

\section{Materials And Methods}

\section{Antibodies and Reagents}

The peptide Tat-CX3CL1 (357-395aa) (catalog number: 04010055093, China peptides company, Shanghai, China) was dissolved in DMSO. The peptide sequence is followed: 5-FITC-(Acp) MFAYQSLQGCPRKMAGEMVEGLRYVPRSCGSNS YVLVPV (purity >95\%). Primary antibodies included: Rabbit polyclonal anti-lba1 (catalog number: 019-19741, Wako company, Japan, 1:500), rat monoclonal anti-CD68 (catalog number: ab53444, Abcam company, Cambridge, UK, 1:500), Rabbit polyclinal to Myelin Basic Protein antibody \catalog number: ab40390, Abcam company, Cambridge, UK, 1:500』, mouse anti-Neurofilament H (NF-H) nonphosphory-lated antibody (SMI32; catalog number: 801701, BioLegend, San Diego, CA, USA,1:500). The secondary antibodies were followed: Alexa Flour cy3 goat 
anti-rabbit IgG (catalog number: E031620, Earthox, LLC, San Francisco, CA, USA, 1:500), Alexa Flour 488 donkey anti-rat IgG (catalog number:21208, Invitrogen, 1:000), Alexa Flour 488 goat anti-mouse IgG (catalog number: ab150113, Abcam company, Cambridge, UK, 1:1000). ELISA kits were purchased from R\&D. GW280264x (catalog number: 555806, Medkoo Biosciences).

\section{Animals}

Two hundred and sixty-one male C57BL/6 mice (22-26 g weight) were purchased from Jinan Pengyue Company (Shandong, China). Mice were housed in a $12 \mathrm{~h} / 12 \mathrm{~h}$ light-dark cycle with ad libitum food and water. The animal procedures were approved by the University Committee on Animal Care of Xuzhou Medical University (ethics No. 201702w012). And animal experiments were complied with the ARRIVE

guidelines and carried out in accordance with the National Institutes of Health guide for the care and use of laboratory animals. Due to the protection of estrogen on ischemic injury and stress, only male mice were used in this study. Mice were euthanized by cervical dislocation and followed the study design as shown in Fig 1.

\section{Middle Cerebral Artery Occlusion Model (MCAO)}

The surgery protocol followed the previous mentioned [18]. Mice were induced anesthesia with $3 \%$ isoflurane in a $30 \% \mathrm{O}_{2} / 68.5 \% \mathrm{~N}_{2} \mathrm{O}$ mixture and were maintained anesthesia $1.5 \%$ isoflurane in a $30 \%$ $\mathrm{O}_{2} / 68.5 \% \mathrm{~N}_{2} \mathrm{O}$ mixture. Middle cerebral artery occlusion (tMCAO) model was performed for study. $\mathrm{A}$ median incision was made in the neck to separate the right common carotid artery (CCA), right internal carotid artery (ICA) and right external carotid artery (ECA). Silica gel thread plug (catalog number: 9070001201, RWD company, Shenzhen, China) was inserted into the right middle cerebral artery of mice. After 60 minutes of ischemia, the silica gel thread plug was slowly pulled out to start reperfusion. The stump of blood vessel was ligated, and the skin was sutured with 4-0 suture (Chenghe suture, Ningbo, China). The mice were placed in the incubator until they fully woke up, and then placed in the breeding box. During the surgery, mice rectal temperature was maintained at $37 \pm 0.5^{\circ} \mathrm{C}$. In the sham operation group, mice received the same surgical procedures without occluding the carotid arteries.

The mice limb movement and consciousness would be evaluated after they are fully awake to determine whether to include or exclude. Mice would be included for next experiment with abnormal limb movement and unconscious disturbance. Otherwise, they would be excluded with one of the following conditions: normal movement and no deviation from the left and right; death or unconsciousness onset shortly after surgery; continuous or intermittent jumped or rolled-over body.

To alleviate the mice postoperative pain, we used $3 \mathrm{mg} / \mathrm{kg}$ ketoprofen once a day at least 3 days after surgery via abdominal injection. In addition, to better take care of the post-operative mice, we used glucose and sodium chloride injection twice a day at least 3 days after surgery.

The mortality rate suffering surgery was $8.05 \%$. A total of 261 mice were used in this study, including 26 mice excluded because of death ( 5 mice), cerebral hemorrhage ( 8 mice), disturbance of consciousness ( 8 
mice) or failure of ischemia induction (5 mice) (As show in Fig 1B).

\section{Lateral ventricular injection}

Based on our previous research, 357-395 amino acid sequence of CX3CL1 are necessary for combination with PSD-93 [17]. And the binding between PSD-93 and CX3CL1 promotes the activation of microglia in acute ischemic cerebral infarction. Thus, we constructed the small peptide CX3CL1 (357-395aa) to disturb combination of PSD-93 and CX3CL1 [17]. Tat-CX3CL1 (357-395aa) were diluted with DMSO at $2.5 \mu \mathrm{g} / \mu \mathrm{L}, 5 \mu \mathrm{g} / \mu \mathrm{L}$, and $10 \mu \mathrm{g} / \mu \mathrm{L}$ and were injected in intracerebroventricular injection with $2 \mathrm{ul}$ per mouse. Mice received DMSO or peptide randomly half an hour before MCAO and repeated at $1 \mathrm{~d}, 2 \mathrm{~d}$, and $3 d$ postsurgery. The right cerebral ventricle was selected to inject the peptide or DMSO (from the bregma: anteroposterior-1 mm; lateral $1 \mathrm{~mm}$; depth $2 \mathrm{~mm}$ ) [17].

\section{Intranasal GW280264x administration}

The ADAM17 inhibitor GW280264x was treated using intranasal[ $[18,19]$ and four drug concentration $(0.25 \mu \mathrm{g} / \mu \mathrm{l}, 0.5 \mu \mathrm{g} / \mu \mathrm{l}, 1.0 \mu \mathrm{g} / \mu \mathrm{l}$, and $1.5 \mu \mathrm{g} / \mu \mathrm{l})$ was set based on previous reported. Mice were randomly assigned to receive DMSO or GW280264x nanoparticle treatment 30 min before MCAO surgery. Five 2- $\mu \mathrm{L}$ drops (total $10 \mu \mathrm{L}$ ) of GW280264x were applied alternately into each nostril with a 2-min interval between drops. Control groups received the same volume of DMSO.

\section{Triphenyl tetrazolium chloride (TTC) staining}

TTC staining was performed as described previously[15] . On the 7th day after surgery, the mice were anesthetized and decapitated directly, and the brain tissue was placed in the mice brain mold (reward). The brains were rapidly frozen at $-80^{\circ} \mathrm{C}$ for about $5 \mathrm{~min}$ and cut one piece every $1 \mathrm{~mm}$. The first knife is at the midpoint of the line between the anterior pole and the optic chiasm and total 5-6 slices were stained with $2 \%$ TTC (2,3,5-triphenyltetrazolium chloride) (Catalog No.BCBW4269, sigma company, USA) at $37^{\circ} \mathrm{C}$ for $15 \mathrm{~min}$ in the dark. The normal tissue was red and the infarcted tissue was white. Filter paper was used to absorb the surface dye of brain slices, and $4 \%$ paraformaldehyde was used for internal fixation overnight. The infarct area ratio was analyzed by image pro Plus6.0 software. Infarct volume ratio $(\%)=\mathrm{V} 1 / \mathrm{V} 2 \times 100 \%, \mathrm{~V} 1=\sum \mathrm{S} 1 \times \mathrm{d}, \mathrm{V} 2=\sum \mathrm{S} 2 \times \mathrm{D}(\mathrm{S} 1$ : infarct area of each section; $\mathrm{S} 2$ : total area of each section; $\mathrm{d}$ : thickness of each piece is $1 \mathrm{~mm}$ ).

\section{Quantitative PCR Assay}

The quantitative real-time PCR methods were carried out as described previously[16]. Total RNA was extracted using Trizol reagent (Invitrogen, Carlsbad, CA, USA, Cat. 15596-026) and reverse transcribed to coding deoxyribonucleic acid (cDNA) using a cDNA synthesis kit (catalog number: RR037A, TAKARA company, Japan). The reaction conditions were $95^{\circ} \mathrm{C}$ for $10 \mathrm{~min}$, followed by 40 cycles at $95^{\circ} \mathrm{C}$ for $15 \mathrm{~s}$ and $60^{\circ} \mathrm{C}$ for $60 \mathrm{~s}$. The following primers were purchased from SANGON Biotech (Shanghai, China) (see in Table 1). The relative expression of target genes was calculated using the $2^{-\triangle \Delta C T}$ method, with 
glyceraldehyde 3-phosphate dehydrogenase (GAPDH) as an internal control. Data were expressed as fold change in expression relative to sham-operated mouse group.

\section{Immunofluorescent Assay}

The detail procedure was reported previously [18]. The mice were euthanized and fixed with $4 \%$ paraformaldehyde through cardiac perfusion. The mice brains were removed and further fixed in $4 \%$ paraformaldehyde at $4{ }^{\circ} \mathrm{C}$ for 24 hours. Brain tissue was treated with $30 \%$ sucrose solution for 72 hours for dehydration. The brain slices ( $20 \mu \mathrm{m}$ thick) were cut with a Leica cryo microtome and kept cryostorage with the antifreeze solution. Sections were blocked with $5 \%$ BSA (solution with $0.3 \%$ Triton X-100 in PBS, PBST) (Catalog No. 28020, Solarbio, Beijing, China) at room temperature for 1 hour and then incubated with primary antibodies at $4{ }^{\circ} \mathrm{C}$ overnight. After washing 3 times with PBST, the sections were incubated for 1 hour in room temperature with specific fluorescent second antibodies. After washing 3 times with $0.3 \%$ Triton X-100 in PBS solution, specific fluorescent secondary antibodies were incubated at room temperature for 1 hour. We used confocal microscopy to capture images and selected two fields in peri-infarct area in each section. And three typical sections having infarct site for each mouse were used to assess.

\section{Evaluation of brain water content}

Brain water content was measured with a common wet-dry weight method [20]. Mice were euthanized and brains were removed carefully and quickly. Then we obtained the wet weight (WW) after removing olfactory bulbs, cerebellum and pons. Subsequently, dry weight (DW) were obtained after the brains were put in an oven at $110^{\circ} \mathrm{C}$ for $6 \mathrm{~h}$ to dry. Brain water content was evaluated by formula: $\mathrm{WC}=$ $(W W-D W) / W W \times 100 \%$.

\section{Behavioral tests}

Modified Neurological function score (mNSS). Based on motor, sensory, reflex, and balance tests, neurological function were assessed with modified Neurological Severity Scores (mNSS), as described previously[15]. Total mNSS score were 18 points, and the higher the score, the more severe the neurological impairment. And we evaluated at $1 \mathrm{~d}, 3 \mathrm{~d}, 5 \mathrm{~d}$ and $1 \mathrm{w}$ after stroke, respectively.

Morris water maze test. Morris water maze test was used to analyze the cognition function including learning and memory ability following with our previous described[18]. Animals were trained on three trials one day for three consecutive days before MCAO. And the experiment was carried out at 31-34 $d$ after MCAO surgery. The water maze device is a circular pool (diameter $=121 \mathrm{~cm}$ ) with opaque water and dark gray wall and bottom and the depth is $45 \mathrm{~cm}$. A square platform (diameter $=10 \mathrm{~cm}$ ) was submerged $1 \mathrm{~cm}$ beneath the water surface and located in the second quadrant of the water maze device.

Furthermore, the water temperature was kept at $21^{\circ} \mathrm{C}$. In the learning test, mice were placed into the pool from one of the four quadrants respectively and allowed to find the hidden platform for $60 \mathrm{~s}$. If the mice did not achieve the platform within $60 \mathrm{~s}$, the experiment finished and the mouse was guided to the 
platform with a wooden stick and stayed on the platform for $10 \mathrm{~s}$ to learn and form the memory of the location of the platform, and then the mouse was removed for the next experiment. Each animal was trained 4 times a day with an interval of 10-15 min for 3 consecutive days. The time spent to reach the platform was recorded. The memory test was performed on 34 day. And the platform in the second quadrant was removed and the behavioral software was used to record the time of mice in the target quadrant (the quadrant where the platform was originally placed), the number of times of entering the quadrant platform and the total distance of swimming in $1 \mathrm{~min}$.

Open field test. The test was used to evaluate the state of depression in mice by using the rodent's exploration behavior of the new environment and performed on 28 days after stroke[21]. The equipment was mainly composed of a square open box of $50 \mathrm{~cm} \times 50 \mathrm{~cm} \times 50 \mathrm{~cm}$ and was divided into peripheral area and central area. A video monitor was placed above the open field equipment to record the frequency and residence time of mice entering the central region. One week before the experiment, mice were placed in the behavioral test room for 3-5 min every day, so as to eliminate the fear of the environment and the experimenter. At the beginning of the experiment, mice were gently placed into the open field from the edge, allowing them to explore freely in the open field for $5 \mathrm{~min}$. Recorded the activity time and entry times in the central area of the open field. During the experiment, the investigator should be 1 meter away from the equipment to eliminate the influence on the experimental animals. Once finished, the equipment was cleaned and the labyrinth was wiped with $75 \%$ alcohol to eliminate the influence of animal odor on subsequent experimental animals.

Elevated plus maze. The test was used to evaluate the anti-anxiety behavior by using the contradictory tendency of mice to explore new environment and the fear of the open arm hanging high and performed on 29 days after stroke[22]. It was used to allow the mice acclimatize the facility for at least 7 days before experiment. The elevated cross maze consists of two open arms with $50 \mathrm{~cm}$ long and $10 \mathrm{~cm}$ wide and closed arms. Both sides of the closed arms are covered by a plastic plate with a height of $40 \mathrm{~cm}$, while the open arms are not covered by plastic plates. The four arms were connected by a central platform with a $10 \mathrm{~cm} \times 10 \mathrm{~cm}$ open part. And the maze is $50 \mathrm{~cm}$ high from the ground. A video monitor is installed above the maze to record the movement of the animals. The mice were placed on one side of the maze with open arms at the beginning of the experiment, and each animal was placed in the same position thereafter. The number of entries and the time spent in each arm were recorded for 5 minutes. During the experiment, the investigators should be $1 \mathrm{~m}$ away from the maze to eliminate the influence on the experimental animals. After recording, the maze was cleaned and wiped with $75 \%$ alcohol to eliminate the effects of animal odor on subsequent experimental animals.

\section{ELISA}

Soluble CX3CL1 levels were analyzed by ELISA kit (R\&D Systems, Stillwater, MN, USA) according to the manufacturer's instructions.

\section{Statistical analysis}


In this study, the sample sizes per group were obtained by power analyses based on previous studies and the literature [15]. And blind method was used in the whole experiment design and implementation process. For example, the person who assessed the mNSS score or analysis the data did not know the groups.

Data were presented as mean \pm standard error of the mean (SEM). GraphPad Prism software (version 8.1.0, La Jolla, CA, USA) was used to analysis the data which coming from at least 5 mice. Firstly, we analyzed whether the data conform to normal distribution using Kolmogorov-Smirnov test. Student's $t$ test was used to analyze data for continuous variables with normal distributions and Mann-Whitney $U$ rank sum test was used with non-normal distributions. Comparison of differences in means between multiple groups were analyzed using one-way ANOVA. And post hoc Bonferroni tests were used to further test pairwise comparisons when the ANOVA showed significant differences. In all analyses, $P<0.05$ was considered statistically significant.

\section{Results}

\section{Tat-CX3CL1 (357-395aa) reduced ischemic brain injury and improved neurobehavioral function after stroke.}

Our previous study showed that PSD-93, acting as a scaffold protein in the postsynaptic membrane, can mediate ischemic brain injury by binding to the 357-395 amino acid sequence of CX3CL1 through its 420535 amino acid sequence[17]. And the constructed peptide Tat-CX3CL1 (357-395aa) blocking the combination between PSD-93 and CX3CL1 protected ischemic brain injury[17]. But the underlying mechanism needs to further explore.

Consistent with our previous results, we found that Tat-CX3CL1 (357-395aa) displayed neuroprotection in ischemic cerebral infarct. In this study, TTC staining was used to detect the effects of different concentrations of interfering peptides on the volume of cerebral infarction at the time point of $7 \mathrm{~d}$ after cerebral infarction, and the optimal concentration was selected for subsequent experimental study. As shown in Fig 2A, and 2B, comparing with I/R group and DMSO group, Tat-CX3CL1 (357-395aa) both at $5 \mu \mathrm{g} / \mu \mathrm{L}$ and $10 \mu \mathrm{g} / \mu \mathrm{L}$ of concentration significantly reduce the tissue loss caused by stroke and the difference was statistically significant $(P<0.001)$. Furthermore, the reduction of infarct volume was more obvious at $10 \mu \mathrm{g} / \mu \mathrm{L}$ concentration, which was consistent with our previous report. And $10 \mathrm{ug} / \mathrm{uL}$ concentration was selected to further study the function and mechanism of Tat-CX3CL1 (357-395aa). And more, Tat-CX3CL1(357-395aa) improved significantly the mNSS scores at I/R 1d and 3d significantly (as shown in Fig $2 \mathrm{C} \otimes P<0.05$ ).

Tat-CX3CL1 (357-395aa) affects the microglia M1 to M2 phenotype polarization shift in the acute stroke phase.

CX3CL1/CX3CR1 axis regulates microglial activation and function and exerts neuroprotective or neurotoxic effects by mediating the release of inflammatory cytokines in the in the central nervous 
system (CNS) [22-28]. To explore the potential cellular mechanism of Tat-CX3CL1 (357-395aa) in ischemic stroke, we first detected the expression of $\mathrm{M} 1 / \mathrm{M} 2$ type inflammatory factors at different time points after ischemia/reperfusion. The results showed that M1 type inflammatory factors (TNF-a, COX-2, and CCL-2) increased from $6 \mathrm{~h}$ after stroke and peaked at $24 \mathrm{~h}$ (as shown in supplemental 1B, 1D, and 1E). And the expression of other cytokines including iNOS and IL-1 $\beta$ also peaked at $24 \mathrm{~h}$ after stroke (as shown in supplemental 1A and 1C). However, M2 type inflammatory factors (IL-4, CD-163, IL-10, and VEGF) decreased from $3 \mathrm{~h}$ after stroke and mostly maintained to $24 \mathrm{~h}$ (as shown in supplemental 1F-11). And CD163 and IL-10 began to rise from 48h after stroke (as shown in supplemental 1G, $1 \mathrm{H}$ ). Thus, we selected $24 \mathrm{~h}$ time point to further investigate the microglia polarization.

To elucidate the effect of Tat-CX3CL1 (357-395aa) on microglia different polarization in post stroke, the quantitative real-time PCR analysis was performed to detect the $\mathrm{M} 1$ and $\mathrm{M} 2$ type cytokines. As shown in Fig 3A-E, Tat-CX3CL1 (357-395aa) inhibited proinflammatory factors (iNOS, TNF-a, IL-13, COX2 and CCI2) related to $\mathrm{M} 1$ type and facilitated the decreased expression of type anti-inflammatory responses (IL-4, IL10, CD163, and VEGF) related to M2 type at $24 \mathrm{~h}$ after stroke (Fig 3F-I). To further confirm the result, we used immunofluorescence assay to observe the number of M1-type microglia in the cortex and striatum around the infarct area after $24 \mathrm{~h}$ reperfusion and found that the number of positive M1 microglia cells decreased significantly in Tat-CX3CL1 (357-395aa) groups not only in cortex region but also in striatum region (Fig 4A-F). These data suggest a potential new protective target of Tat-CX3CL1 (357-395aa) on microglia M1/M2 phenotype switch during ischemic stroke.

\section{Tat-CX3CL1 (357-395aa) inhibited generation of sCX3CL1 and improved brain edema.}

CX3CL1 is localized on neuronal cells and exists in the form of membrane-anchored CX3CL1 (mCX3CL1) and soluble CX3CL1 (sCX3CL1) isoforms[29]. And once mCX3CL1 is cleaved into SCX3CL1 by the disintegrin and metalloproteinase (ADAM 10 and ADAM 17) and the latter could bind to its only receptor CX3CR1 expressed in microglia and initiates its downstream signaling pathway[30, 31]. Our previous study showed that PSD-93 binds CX3CL1 through their specific amino acid sequence, which promotes membrane-bound CX3CL1 (mCX3CL1) cleave into soluble CX3CL1 (sCX3CL1)[17].

To further elusive the mechanism of peptide Tat-CX3CL1 (357-395) on microglia polarization transformation, we tested the generation of SCX3CL1 and found that Tat-CX3CL1 (357-395) inhibit the formation of SCX3CL1 (as shown in Fig 2F). Meanwhile, we applied GW280264x, an inhibitor of disintegrin and a metalloproteinase ADAM 17, and selected I/R $6 \mathrm{~h}$ time point and observed that GW280264x inhibited the expression of proinflammatory cytokines including iNOS, TNF- $a$, and IL-1 $\beta$ and facilitated the expression of anti-inflammatory cytokines including CD-163, IL-10, and VEGF (as shown in Fig 5).

Ischemic cerebral injury destructed the blood-brain barrier integrity and induced brain edema especially 24-72h after stroke. Tat-CX3CL1 (357-395aa) could also reduce the cerebral edema after infarction remarkably (Fig 2D, 2E). These results indicated that Tat-CX3CL1 (357-395) may decrease the expression 
of mCX3CL1 through blocking the combination of PSD-93 and CX3CL1 and then reverse the proinflammatory state of microglia.

\section{Tat-CX3CL1 (357-395aa) treatment promotes functional recovery of cognitive dysfunction after stroke through improving integrity of myelinated fibers.}

White matter injury after stroke is associated with cognitive deficits and involved with neuroinflammation, demyelination, and degeneration of axons[32-34]. To further explore whether Tat-CX3CL1 (357-395aa) improve the cognitive dysfunction, Morris water maze was designed to investigate mice study and memory function. As shown in Fig 6, Tat-CX3CL1 (357-395aa) reduced the escape latency and extended the time spent in the target quadrant. However, Tat-CX3CL1 (357-395aa) did not affect mice swimming speed (Fig 6C). Moreover, neuroinflammation induced by ischemic cerebral damage may be facilitate the loss of myelin sheath and white matter lesions[35].

In light of above results, we evaluated whether Tat-CX3CL1 (357-395aa) improve white matter integrity in stroke and used dual staining for SMI32 (a marker of demyelinated axons) and myelin basic protein (MBP, a major myelin protein) to assess the lesion in white matter (Fig 6E,6F). The immunofluorescence intensity of MBP staining decreased in corpus callosum (CC) and cortex regions at 35d after MCAO (Fig 6G, 6H). However, Tat-CX3CL1 (357-395aa) increased the loss of myelin protein significantly. SMI32/MBP ratio was used to analyze the repair degree of myelin sheath and white matter. Results showed that in corpus callosum (CC) and cortex regions SMI32/MBP ratio increased in I/R and DMSO group significantly comparing with sham group and lower SMI32/MBP ratio in Tat-CX3CL1 (357-395aa) group comparing with I/R and MDSO group (Fig 6l, 6J). These results suggested that Tat-CX3CL1 (357-395aa) promote the repair of myelin sheath and the recovery of white matter in cortex and CC.

\section{Tat-CX3CL1 (357-395aa) improves poststroke anxiety and depression.}

Post-stroke depression and anxiety exacerbate cognitive dysfunction and the underlying mechanism might be related to microglia polarization. Here, we observed the anxiety-like behavior and depression state using open field test and elevated plus maze. Elevated plus maze were performed to exam anxiety status in mice on 29 days after ischemic stroke. The elevated cross maze included open arm and closed arm and the number of entering the open arm and dwell time were recorded and analysed. As shown in Fig 7, compared with I/R group and DMSO group, the number of entering the open arm and dwell time increased remarkedly. It is suggested that Tat-CX3CL1 (357-395aa) can improve anxiety behavior after ischemic brain injury in mice.

Additionally, we also recorded the number of entering the central region and residence time in open field test and found that Tat-CX3CL1 (357-395aa) increased the frequency of entering the central area and the activity time in the central area of open field significantly. It is suggested that Tat-CX3CL1 (357-395aa) improves post-stroke depression.

\section{Discussion}


The present study identified the robustly protective roles of the Tat-CX3CL1 (357-395aa) through blocking the interaction of PSD-93 and CX3CL1 and reduced the production of soluble CX3CL1, resulting in inhibiting the communication between neuron and microglia. Here, we found that the expression peaks of pro-inflammatory cytokines (M1 like) and anti-inflammatory cytokines (M2 like) were different and peak of $\mathrm{M} 1$ like cytokines was earlier than that of M2 like cytokines. In addition, the peak of SCX3CL expression was the same time point as the starting time of M1 phenotypic microglia activation, which confirm the SCX3CL-CX3CR1 signal pathway mediating the neuron-microglia crosstalk. The peptide TatCX3CL1 (357-395aa) reduced pro-inflammatory cytokines secretion in acute ischemia-reperfusion meanwhile prompting anti-inflammatory cytokines expression (as shown in Fig 8). Furthermore, the peptide Tat-CX3CL1 (357-395aa) diminished the neurological impairment and improved the long-term cognitive dysfunction after stroke. Collectively, these data support the beneficial effects of Tat-CX3CL1 (357-395aa) and the peptide treatment might be a therapeutic potential after ischemic stroke.

Accumulating evidences showed that microglia are polarized into different states within hours following stroke onset. Differential polarization of microglia including classic pro-inflammatory type (M1-like) and alternative protective type (M2-like) is activated at different stage, which exerts detrimental or beneficial potentials[10, 36, 37]. Thus, future therapies targeting microglia not only exclusively focuses on inhibiting microglia activation, but also adjusts its polarization toward M1or M2 phenotype. However, the exact molecular mechanism mediating microglia polarization under ischemic environment needs to further elucidate.

In this study, we found that M1/M2-like microglia activation at different stage after stroke. M1-like microglia was activated at $6 \mathrm{~h}$ after stoke firstly and peaked at $24 \mathrm{~h}$ and then persisted several days. This result is consistent with the peak expression of s CX3CL1 [17], which indicates that splicing into SCX3CL1 promotes M1-like microglia activation. Conversely, M2-like microglia was suppressed at first $24 \mathrm{~h}$ following stroke onset. Meanwhile, we found an interesting phenomenon that Tat-CX3CL1 (357-395aa) facilitates microglia polarization from M1 to M2 phenotype through inhibiting the M1-phenotype cytokines (iNOS, TNF-a, IL-1 $\beta$, COX-2, and CCL-2) and promoted the M2-phenotype cytokines (IL-4, CD163, IL-10, and VEGF) at $24 \mathrm{~h}$ after stroke. This gave us a new target for treatment acute ischemic stroke injury. But the potential mechanisms and its association with behavioral and cognitive dysfunction induced by stroke are still further investigation. And the role of dynamic changes of inflammatory response in subacute cerebral infarction is still worthy of further study.

Our previous intriguing study found that PSD-93 binds with CX3CL1 and their binding amino acid sequence located at 420-535 amino acid sequence of PSD-93 and 357-395 amino acid sequence of CX3CL1[17]. The peptide Tat-CX3CL1 (357-395aa) blocked the binding of PSD-93 and CX3CL1 in the cerebral infarct volume, but the underlying mechanism is still unclear. Microglia plays a vital role in health and disease through CX3CL1/CX3CR1 signaling, which is critical for microglia-neuron cross-talk on the account of their specific cellular localization [24-28, 38]. In addition, PSD-93 combined with CX3CL1 and facilitated it cracking into soluble forms, and then bond to CX3CR1 receptor expressed on microglia [17]. Here, we also found that Tat-CX3CL1 (357-395aa) reduced the release of soluble CX3CL1 and inhibited 
pro-inflammatory type (M1-like) cytokines, which implies that Tat-CX3CL1 (357-395aa) suppressed the soluble CX3CL1 expression and then inhibited CX3CL1/CX3CR1 signaling pathway activation.

Previous study revealed that CX3CL1-CX3CR1 signaling regulated synaptic plasticity and cognitive functions in adult brain[39-42]. Ischemic stroke impacts not only grey matter but also white matter and induces poststroke cognitive impairment deficits both memory and learning. Furthermore, incidence rate of post-stroke anxiety and post-stroke depression was about $36.7 \%$ within 2 weeks after stroke[43, 44], which accounts for detrimental influences on poststroke cognitive impairment. Aside from the effect of microglia polarization transformation, Tat-CX3CL1 (357-395aa) contributed to the white matter repair and cognitive improvement. Additionally, Tat-CX3CL1 (357-395aa) performed a beneficial potential on poststroke anxiety and depression state. These results suggest that inhibition of microglial polarization in acute stage of ischemic infarction will be beneficial to the rehabilitation of nerve function in the later stage. And Tat-CX3CL1 (357-395aa) is attractive for stroke therapies based on their immunoregulation and protective function.

Many evidences suggest that microglia play the biphasic role in cerebral ischemia because of its differential polarization[10, 36, 37]. Single target M1-like microglia is insufficient because microglia polarization is dynamically changing that is new prospects in brain repair. Our study has uncovered a role of Tat-CX3CL1 (357-395aa) in immunoregulation about microglia polarization transformation.

Furthermore, we have indicated that Tat-CX3CL1 (357-395aa) promotes white matter repair and improves poststroke cognitive impairment and poststroke emotional dysfunction. Therefore, it might be a new potential therapeutic strategy for functional recovery after ischemic stroke onset.

\section{Conclusion}

In this study, we revealed the mechanism of PSD-93 regulating CX3CL1/CX3CR1 axes during ischemic stroke. Our findings indicated that Tat-CX3CL1 (357-395aa) inhibited formation of soluble CX3CL1 and promote M2 type microglia polarization, which eventually plays a neuroprotective role in mice ischemic stroke model. To sum up, the peptide Tat-CX3CL1 (357-395aa) might a new target for treatment of ischemic stroke.

\section{Abbreviations}

ADAM, A distintegrin and metalloproteinase; BD, Binding-domain; CX3CL1, Chemokine C-X3-C-Motif ligand 1; IL-1 $\beta$, Interleukin 1 $\beta$; MCAO, Middle cerebral artery occlusion; NMDAR, N-methyl-D-aspartic acid receptor; PSD-93, Postsynaptic densitries-93; TNF-a, Tumor necrosis factor-alpha; MCAO, middle cerebral artery occlusion model; mNSS, modified neurological function score; TTC, triphenyl tetrazolium chloride; $\mathrm{I} / \mathrm{R}$, ischemia/reperfusion; BBB, blood-brain barrier.

\section{Declarations}




\section{Acknowledgements}

Not applicable.

\section{Author contributions}

Qingxiu Zhang designed and performed the experiments, and drafted the manuscript. Yun Xu designed the experiments, interpreted the data and edited the manuscript. Liangqun Rong interpreted the data and edited the manuscript. Xiaowei Cao conducted the experiments, analyzed the data, and drafted the manuscript. Hui Yang performed the experiments and analyzed the data. Liping Kong, Junjun Chan and Shiying Lou conducted the experiments and analyzed the data. Xiaomei Liu conceived the experiments, interpreted the data and edited the manuscript. All authors approved the manuscript before submission.

\section{Ethics approval and consent to participate}

All animal experiments were performed at Xuzhou Medical University according to Animal Care and Use Committee of Xuzhou Medical University. Mice were euthanized by cervical dislocation. Ethics approval reference number was 201702w012.

\section{Consent for publication}

Not applicable.

\section{Availability of data and materials}

All data are available upon reasonable request to correspondence author.

\section{Competing interests}

The authors declare that they have no competing interests.

\section{Funding}

This study was supported by the National Natural Science Foundation of China (No. 81671149, 82071304, and 81971179), the Natural Science Foundation of Jiangsu Province (No.BK20161167, and BK20191463), Subject of Xuzhou Science and Technology Bureau (KC19167, KC16SS081 and KC17115), and Youth Project of Health Department of Jiangsu Province (Q201618).

\section{References}

1. Global regional. and national burden of stroke, 1990-2016: a systematic analysis for the Global Burden of Disease Study 2016. Lancet Neurol. 2019;18:439-58.

2. Jin R, Liu L, Zhang S, Nanda A, Li G. Role of inflammation and its mediators in acute ischemic stroke. J Cardiovasc Transl Res. 2013;6:834-51. 
3. Rong R, Yang H, Rong L, Wei X, Li Q, Liu X, Gao H, Xu Y, Zhang Q. Proteomic analysis of PSD-93 knockout mice following the induction of ischemic cerebral injury. Neurotoxicology. 2016;53:1-11.

4. Zhang M, Li Q, Chen L, Li J, Zhang X, Chen X, Zhang Q, Shao Y, Xu Y. PSD-93 deletion inhibits Fynmediated phosphorylation of NR2B and protects against focal cerebral ischemia. Neurobiol Dis. 2014;68:104-11.

5. Ma Y, Wang J, Wang Y, Yang GY. The biphasic function of microglia in ischemic stroke. Prog Neurobiol. 2017;157:247-72.

6. Wang J, Xing $\mathrm{H}$, Wan $\mathrm{L}$, Jiang $\mathrm{X}$, Wang $\mathrm{C}$, Wu Y. Treatment targets for $\mathrm{M} 2$ microglia polarization in ischemic stroke. Biomed Pharmacother. 2018;105:518-25.

7. Gelderblom M, Leypoldt F, Steinbach K, Behrens D, Choe CU, Siler DA, Arumugam TV, Orthey E, Gerloff $\mathrm{C}$, Tolosa E, Magnus T. Temporal and spatial dynamics of cerebral immune cell accumulation in stroke. Stroke. 2009;40:1849-57.

8. Hu X, Leak RK, Shi Y, Suenaga J, Gao Y, Zheng P, Chen J. Microglial and macrophage polarizationnew prospects for brain repair. Nat Rev Neurol. 2015;11:56-64.

9. Gülke E, Gelderblom M, Magnus T. Danger signals in stroke and their role on microglia activation after ischemia. Ther Adv Neurol Disord. 2018;11:1756286418774254.

10. Hu X, Li P, Guo Y, Wang H, Leak RK, Chen S, Gao Y, Chen J. Microglia/macrophage polarization dynamics reveal novel mechanism of injury expansion after focal cerebral ischemia. Stroke. 2012;43:3063-70.

11. Rayasam A, Hsu M, Kijak JA, Kissel L, Hernandez G, Sandor M, Fabry Z: Immune responses in stroke: how the immune system contributes to damage and healing after stroke and how this knowledge could be translated to better cures? Immunology 2018, 154:363 - 76.

12. Liu X, Liu J, Zhao S, Zhang H, Cai W, Cai M, Ji X, Leak RK, Gao Y, Chen J, Hu X. Interleukin-4 Is Essential for Microglia/Macrophage M2 Polarization and Long-Term Recovery After Cerebral Ischemia. Stroke. 2016;47:498-504.

13. Qin C, Zhou LQ, Ma XT, Hu ZW, Yang S, Chen M, Bosco DB, Wu LJ, Tian DS. Dual Functions of Microglia in Ischemic Stroke. Neurosci Bull. 2019;35:921-33.

14. Fumagalli $M$, Lombardi $M$, Gressens $P$, Verderio $C$. How to reprogram microglia toward beneficial functions. Glia. 2018;66:2531-49.

15. Zhang Q, Yang H, Gao H, Liu X, Li Q, Rong R, Liu Z, Wei XE, Kong L, Xu Y, Rong L. PSD-93 Interacts with SynGAP and Promotes SynGAP Ubiquitination and Ischemic Brain Injury in Mice. TransI Stroke Res. 2020;11:1137-47.

16. Zhang Q, Cheng H, Rong R, Yang H, Ji Q, Li Q, Rong L, Hu G, Xu Y. The Effect of PSD-93 Deficiency on the Expression of Early Inflammatory Cytokines Induced by Ischemic Brain Injury. Cell Biochem Biophys. 2015;73:695-700.

17. Zhang Q, He L, Chen M, Yang H, Cao X, Liu X, Hao Q, Chen Z, Liu T, Wei XE, Rong L. PSD-93 mediates the crosstalk between neuron and microglia and facilitates acute ischemic stroke injury by binding to CX3CL1. J Neurochem 2021. 
18. Zhang Q, Zhu W, Xu F, Dai X, Shi L, Cai W, Mu H, Hitchens TK, Foley LM, Liu X, et al. The interleukin4/PPARy signaling axis promotes oligodendrocyte differentiation and remyelination after brain injury. PLoS Biol. 2019;17:e3000330.

19. Dreymueller D, Martin C, Kogel T, Pruessmeyer J, Hess FM, Horiuchi K, Uhlig S, Ludwig A. Lung endothelial ADAM17 regulates the acute inflammatory response to lipopolysaccharide. EMBO Mol Med. 2012;4:412-23.

20. Qi W, Cao D, Li Y, Peng A, Wang Y, Gao K, Tao C, Wu Y. Atorvastatin ameliorates early brain injury through inhibition of apoptosis and ER stress in a rat model of subarachnoid hemorrhage. Biosci Rep 2018, 38.

21. Donatti AF, Soriano RN, Leite-Panissi CR, Branco LG, de Souza AS. Anxiolytic-like effect of hydrogen sulfide $(\mathrm{H}(2) \mathrm{S})$ in rats exposed and re-exposed to the elevated plus-maze and open field tests. Neurosci Lett. 2017;642:77-85.

22. Kraeuter AK, Guest PC, Sarnyai Z. The Elevated Plus Maze Test for Measuring Anxiety-Like Behavior in Rodents. Methods Mol Biol. 2019;1916:69-74.

23. Laing JM, Smith CC, Aurelian L. Multi-targeted neuroprotection by the HSV-2 gene ICP10PK includes robust bystander activity through PI3-K/Akt and/or MEK/ERK-dependent neuronal release of vascular endothelial growth factor and fractalkine. J Neurochem. 2010;112:662-76.

24. Febinger HY, Thomasy HE, Pavlova MN, Ringgold KM, Barf PR, George AM, Grillo JN, Bachstetter AD, Garcia JA, Cardona AE, et al. Time-dependent effects of CX3CR1 in a mouse model of mild traumatic brain injury. J Neuroinflammation. 2015;12:154.

25. Lee S, Xu G, Jay TR, Bhatta S, Kim KW, Jung S, Landreth GE, Ransohoff RM, Lamb BT. Opposing effects of membrane-anchored CX3CL1 on amyloid and tau pathologies via the p38 MAPK pathway. J Neurosci. 2014;34:12538-46.

26. Pabon MM, Bachstetter AD, Hudson CE, Gemma C, Bickford PC. CX3CL1 reduces neurotoxicity and microglial activation in a rat model of Parkinson's disease. J Neuroinflammation. 2011;8:9.

27. Shan S, Hong-Min T, Yi F, Jun-Peng G, Yue F, Yan-Hong T, Yun-Ke Y, Wen-Wei L, Xiang-Yu W, Jun M, et al. New evidences for fractalkine/CX3CL1 involved in substantia nigral microglial activation and behavioral changes in a rat model of Parkinson's disease. Neurobiol Aging. 2011;32:443-58.

28. Mizuno T, Kawanokuchi J, Numata K, Suzumura A. Production and neuroprotective functions of fractalkine in the central nervous system. Brain Res. 2003;979:65-70.

29. Bajetto A, Bonavia R, Barbero S, Schettini G. Characterization of chemokines and their receptors in the central nervous system: physiopathological implications. J Neurochem. 2002;82:1311-29.

30. Clark AK, Yip PK, Grist J, Gentry C, Staniland AA, Marchand F, Dehvari M, Wotherspoon G, Winter J, Ullah J, et al. Inhibition of spinal microglial cathepsin $S$ for the reversal of neuropathic pain. Proc Natl Acad Sci U S A. 2007;104:10655-60.

31. Garton KJ, Gough PJ, Blobel CP, Murphy G, Greaves DR, Dempsey PJ, Raines EW. Tumor necrosis factor-alpha-converting enzyme (ADAM17) mediates the cleavage and shedding of fractalkine (CX3CL1). J Biol Chem. 2001;276:37993-8001. 
32. Rosenzweig S, Carmichael ST. The axon-glia unit in white matter stroke: mechanisms of damage and recovery. Brain Res. 2015;1623:123-34.

33. Shi H, Hu X, Leak RK, Shi Y, An C, Suenaga J, Chen J, Gao Y. Demyelination as a rational therapeutic target for ischemic or traumatic brain injury. Exp Neurol. 2015;272:17-25.

34. Wang Y, Liu G, Hong D, Chen F, Ji X, Cao G. White matter injury in ischemic stroke. Prog Neurobiol. 2016;141:45-60.

35. Liu Y, Wu C, Hou Z, Fu X, Yuan L, Sun S, Zhang H, Yang D, Yao X, Yang J. Pseudoginsenoside-F11 Accelerates Microglial Phagocytosis of Myelin Debris and Attenuates Cerebral Ischemic Injury Through Complement Receptor 3. Neuroscience. 2020;426:33-49.

36. Mabuchi T, Kitagawa K, Ohtsuki T, Kuwabara K, Yagita Y, Yanagihara T, Hori M, Matsumoto M. Contribution of microglia/macrophages to expansion of infarction and response of oligodendrocytes after focal cerebral ischemia in rats. Stroke. 2000;31:1735-43.

37. Lakhan SE, Kirchgessner A, Hofer M. Inflammatory mechanisms in ischemic stroke: therapeutic approaches. J Transl Med. 2009;7:97.

38. Luo P, Chu SF, Zhang Z, Xia CY, Chen NH. Fractalkine/CX3CR1 is involved in the cross-talk between neuron and glia in neurological diseases. Brain Res Bull. 2019;146:12-21.

39. Meucci O, Fatatis A, Simen AA, Miller RJ. Expression of CX3CR1 chemokine receptors on neurons and their role in neuronal survival. Proc Natl Acad Sci U S A. 2000;97:8075-80.

40. Ragozzino D, Di Angelantonio S, Trettel F, Bertollini C, Maggi L, Gross C, Charo IF, Limatola C, Eusebi F. Chemokine fractalkine/CX3CL1 negatively modulates active glutamatergic synapses in rat hippocampal neurons. J Neurosci. 2006;26:10488-98.

41. Deiva K, Geeraerts T, Salim H, Leclerc P, Héry C, Hugel B, Freyssinet JM, Tardieu M. Fractalkine reduces $\mathrm{N}$-methyl-d-aspartate-induced calcium flux and apoptosis in human neurons through extracellular signal-regulated kinase activation. Eur J Neurosci. 2004;20:3222-32.

42. Limatola C, Lauro C, Catalano M, Ciotti MT, Bertollini C, Di Angelantonio S, Ragozzino D, Eusebi F. Chemokine $\mathrm{CX} 3 \mathrm{CL} 1$ protects rat hippocampal neurons against glutamate-mediated excitotoxicity. $J$ Neuroimmunol. 2005;166:19-28.

43. Pérez-Piñar M, Ayerbe L, González E, Mathur R, Foguet-Boreu Q, Ayis S. Anxiety disorders and risk of stroke: A systematic review and meta-analysis. Eur Psychiatry. 2017;41:102-08.

44. Rafsten L, Danielsson A, Sunnerhagen KS. Anxiety after stroke: A systematic review and metaanalysis. J Rehabil Med. 2018;50:769-78.

\section{Tables}

Table 1 PCR primers sequences of different gene fragments. 


\begin{tabular}{|c|c|}
\hline Name & Primer \\
\hline \multirow[t]{2}{*}{ GAPDH } & Forward: 5'- GCCAAGGCTGTGGGCAAGGT-3' \\
\hline & Reverse: 5'-TCTCCAGGCGGCACGTCAGA-3' \\
\hline \multirow[t]{2}{*}{ TNF-a } & Forward:5'-TGTGCTCAGAGCTTTCAACAA-3' \\
\hline & Reverse: 5'-CTTGATGGTGGTGCATGAGA-3' \\
\hline \multirow[t]{2}{*}{ IL-10 } & Forward:5'-GGCATGAGGATCAGCAGGGGC-3' \\
\hline & Reverse:5'-TGGCTGAAGGCAGTCCGCAG-3' \\
\hline \multirow[t]{2}{*}{ iNOS } & Forward:5'-CAGCTGGGCTGTACAAACCTT-3' \\
\hline & Reverse:5'-CATTGGAAGTGAAGCGTTTCG-3' \\
\hline \multirow[t]{2}{*}{ IL-4 } & Forward:5'-GAATGTACCAGGAGCCATATC-3' \\
\hline & Reverse:5'-CTCAGTACTACGAGTAATCCA-3' \\
\hline \multirow[t]{2}{*}{$\operatorname{cox} 2$} & Forward:5'- CTCTACATAAGCCAGTGAGA -3' \\
\hline & Reverse:5'- TCATCTTGTAACAACACTCAC -3' \\
\hline \multirow[t]{2}{*}{ CCL-2 } & Forward: 5'- GCTGGAGCATCCACGTGTT -3' \\
\hline & Reverse: 5'- ATCTTGCTGGTGAATGAGTAGCA -3' \\
\hline \multirow[t]{2}{*}{ CD163 } & Forward: 5'-GGGAAGAGTGGAGCTCAAGA-3' \\
\hline & Reverse: 5'-ACCAGCTCCTTTCCCAAAAT-3' \\
\hline \multirow[t]{2}{*}{ IL-1 $\beta$} & Forward: 5'-AAGCCTCGTGC TGTCGGACC-3' \\
\hline & Reverse: 5'-TGAGGCCCAAGGCCACAGGT-3' \\
\hline \multirow[t]{2}{*}{ VEGF } & Forward: 5'-GGGGAGCAAGCAAGGCCAGG-3' \\
\hline & Reverse: 5'-TCTCTGCCTCCGTGAGGGGC-3' \\
\hline
\end{tabular}

\section{Figures}


$\mathbf{A}$

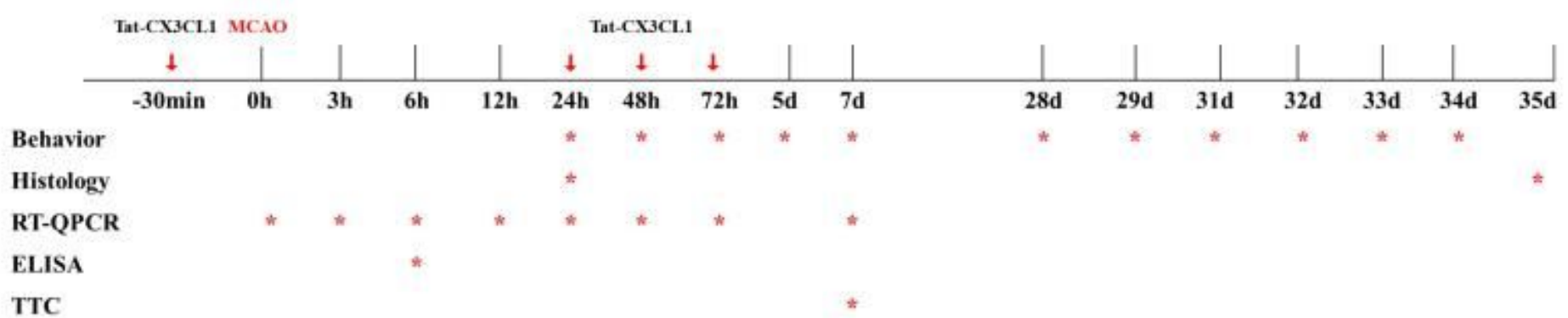

B

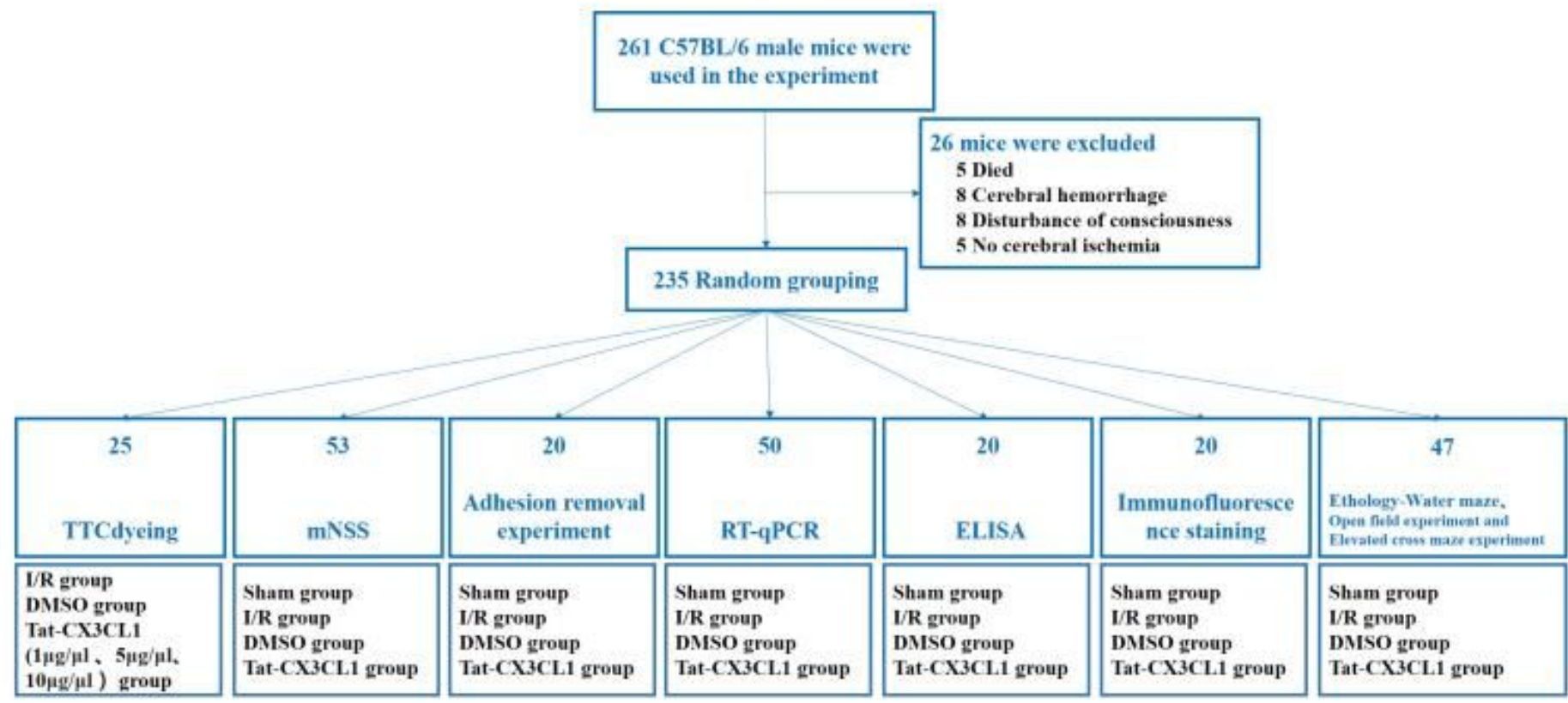

\section{Figure 1}

Study design flowchart. (A) Showed the timeline schematic of the experimental procedure. (B) Showed the flowchart of mice experimental grouping. 
A

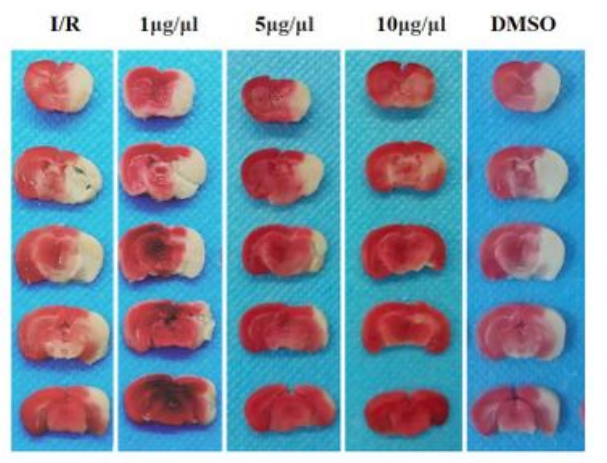

D
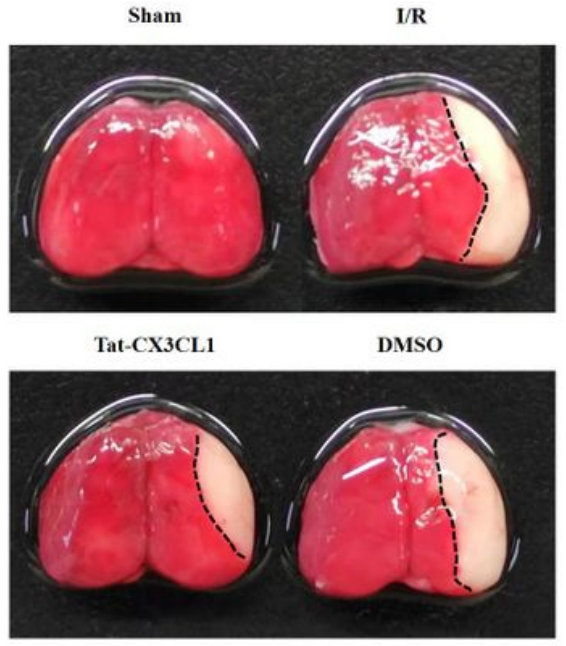

B

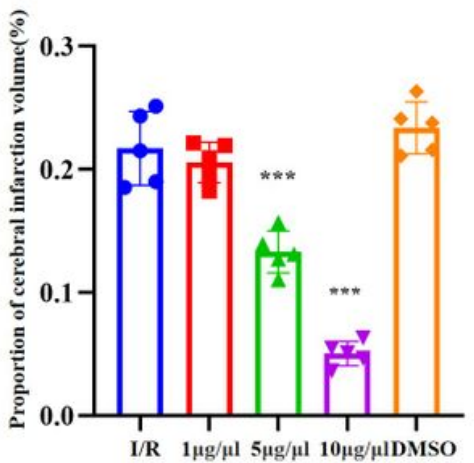

E

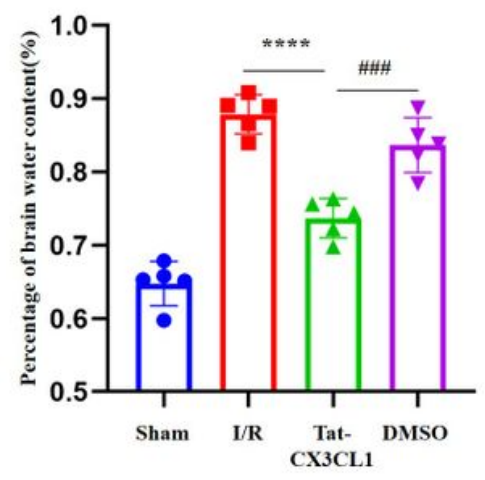

C

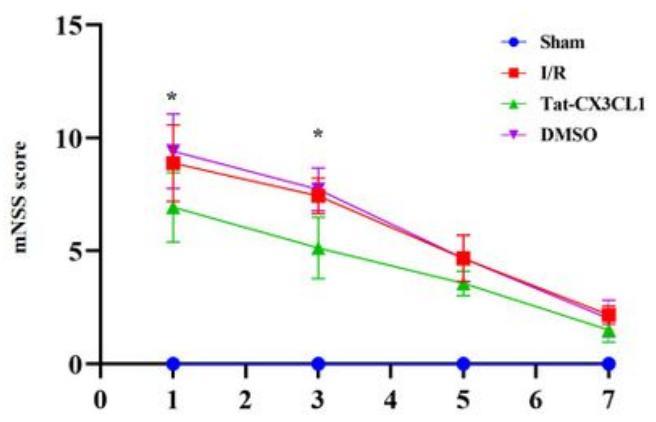

$\mathbf{F}$

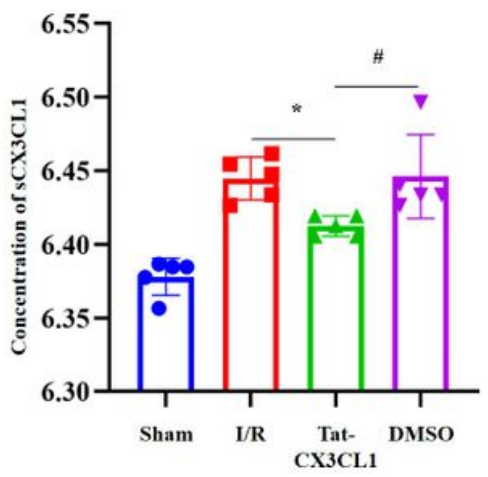

Figure 2

Tat-CX3CL1 (357-395aa) reduced infarct volume, brain edema and improved neurological function in mice after cerebral infarction. (A-B) TTC staining showed that Tat-CX3CL1 (357-395aa) reduced infarct volume. ${ }^{* \star *} \mathrm{P}<0.001,5 \mu \mathrm{g} / \mu \mathrm{L}$ Tat-CX3CL1(357-395aa) group versus DMSO group; \#\#\#P<0.001, 10 $\mu \mathrm{g} / \mu \mathrm{L}$ Tat-CX3CL1(357-395aa) group versus DMSO group, $n=5$. (C) mNSS scores showed a gradual improvement in neurological function after cerebral infarction in Tat-CX3CL1 (357-395aa) group. * $P<0.05$, Tat-CX3CL1 (357-395aa) group versus DMSO group, $n=8-17$. (D) TTC staining of mice brain at R24h in each group. (E) Tat-CX3CL1 (357-395aa) reduced the water content of brain tissue after infarction. $* \star \star \star P$ $<$ 0.0001, Tat-CX3CL1 (357-395aa) group versus I/R group; \#\#\#P<0.001, Tat-CX3CL1 (357-395aa) group versus DMSO group, $n=5$. (F) Tat-CX3CL1 (357-395aa) inhibited the expression of sCX3CL1 at R6h. ${ }^{*} \mathrm{P}<0.05$, Tat-CX3CL1 (357-395aa) group versus I/R group; \#P<0.05, Tat-CX3CL1 (357-395aa) group versus DMSO group, $n=5$. Results are expressed as means \pm SEM. 
A

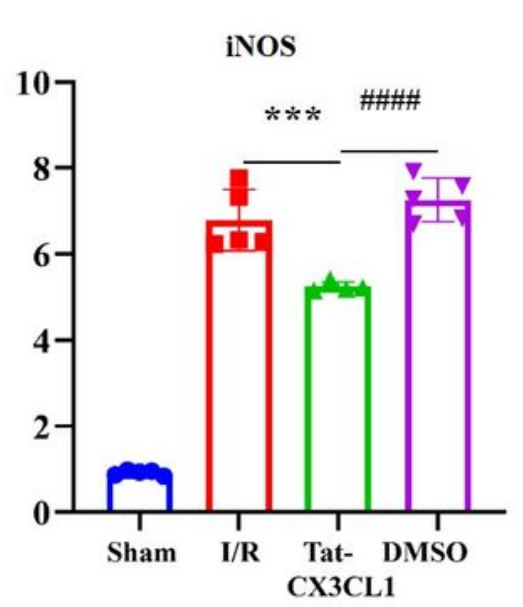

D

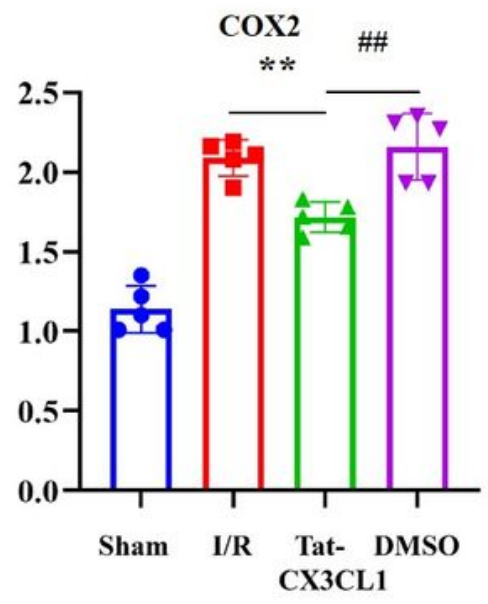

G

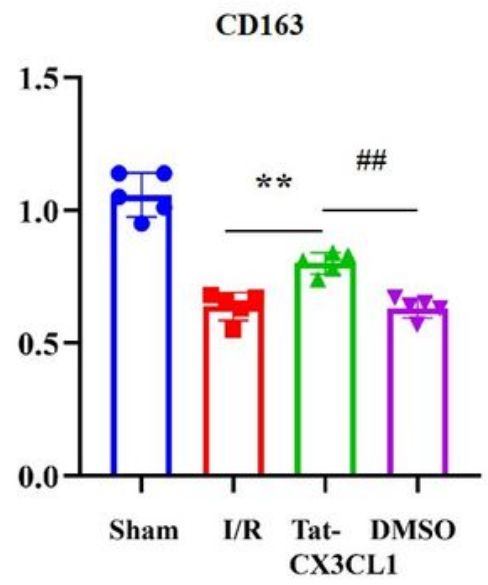

B

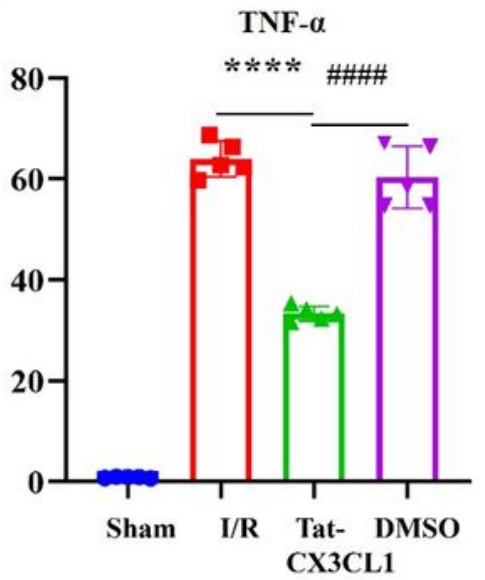

E

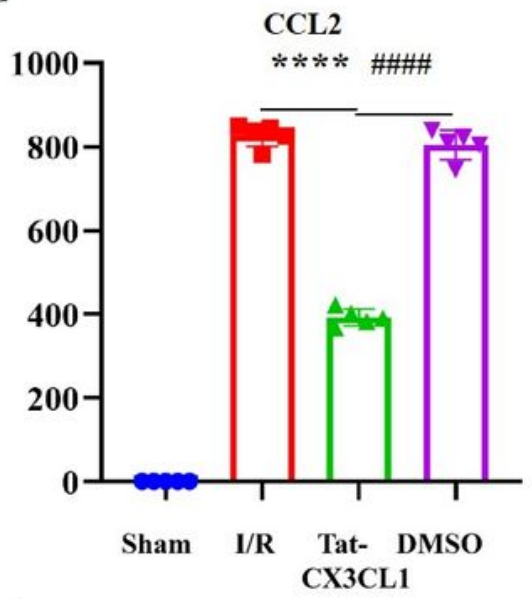

H

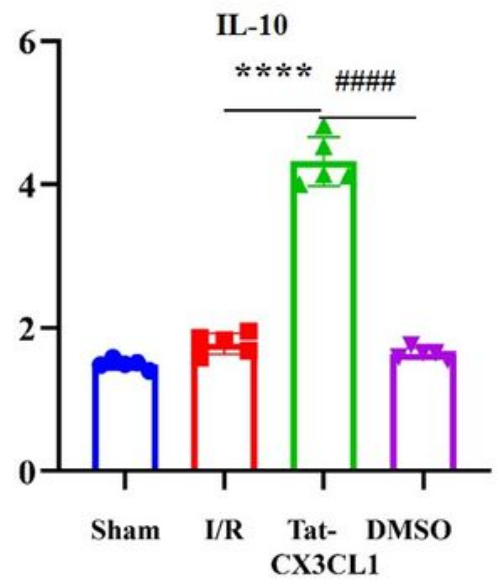

C

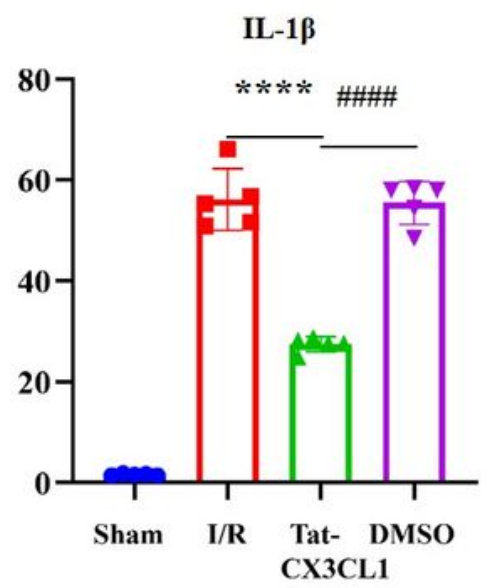

F

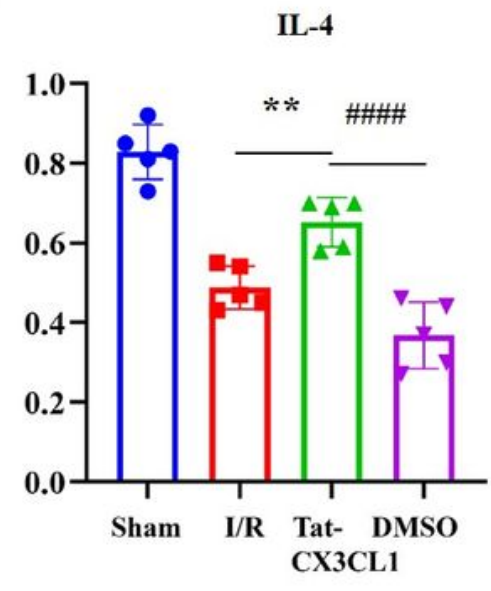

I

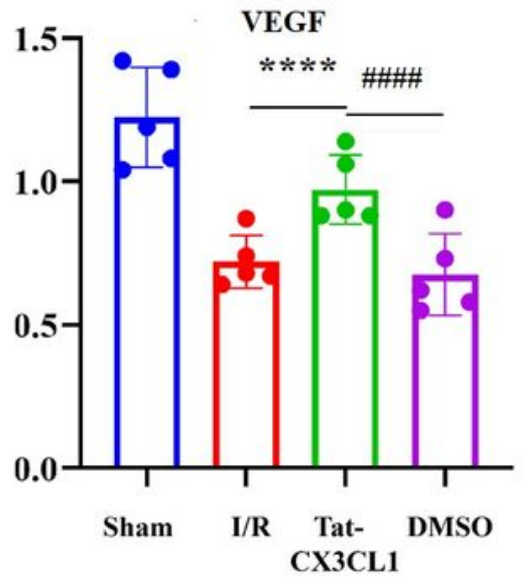

Figure 3

Effects of Tat-CX3CL1 (357-395aa) on phenotypic polarization of M1/M2 microglia at R24h. Effects of Tat-CX3CL1(357-395aa) on phenotypic polarization of M1/M2 microglia at R24h. (A-E) Tat-CX3CL1(357395aa) reduced the expression of iNOS, TNF-a, IL-1ß, COX2 and CCL2. (F-I) Tat-CX3CL1(357-395aa) increased the expression of IL-4, CD163, IL-10 and VEGF. Tat - CX3CL1(357-395aa) group I/R group, *P < 
0.05, ${ }^{\star \star} \mathrm{P}<0.01,{ }^{\star * \star} \mathrm{P}<0.001,{ }^{\star \star * \star *} \mathrm{P}<0.0001$; Tat $-\mathrm{CX} 3 \mathrm{CL} 1$ group versus DMSO group, \#P $<0.05$, \#\#P $<$ 0.01, \#\#\#P $<0.001$, \#\#\#\#P $<0.0001, n=5)$. Results are expressed as means \pm SEM.

A

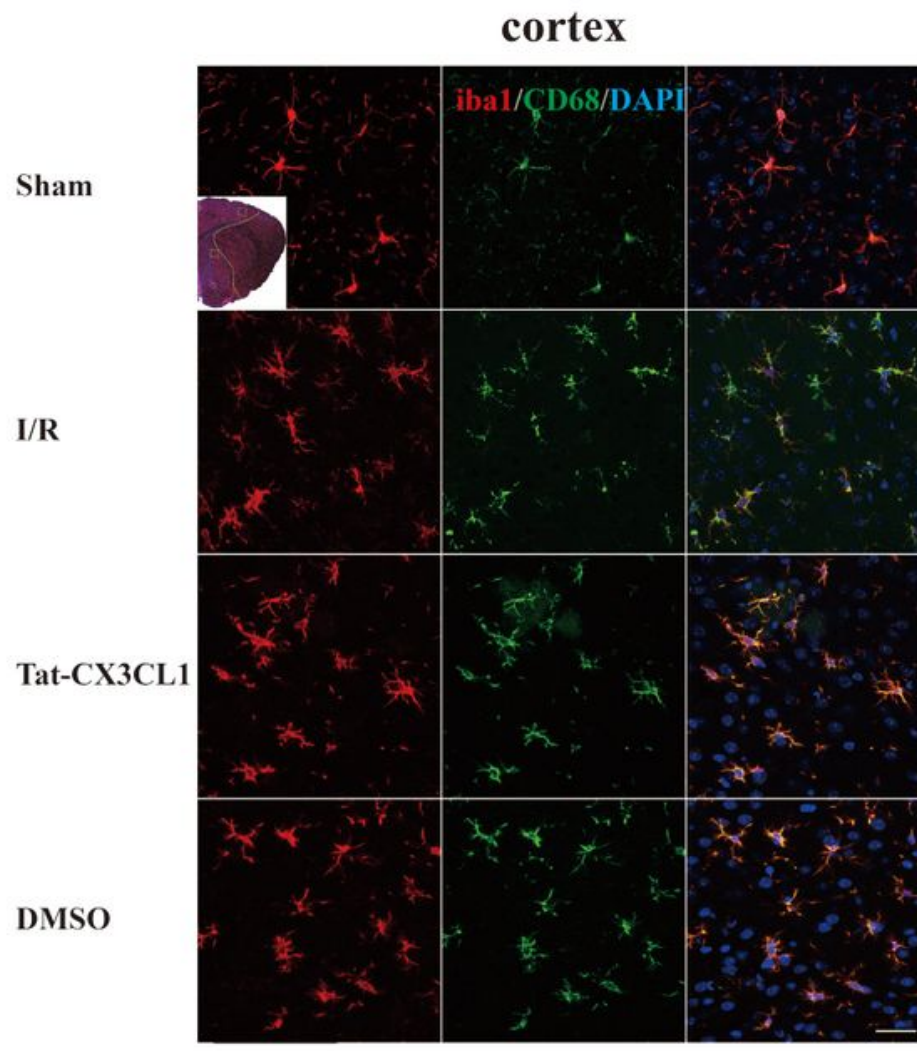

B

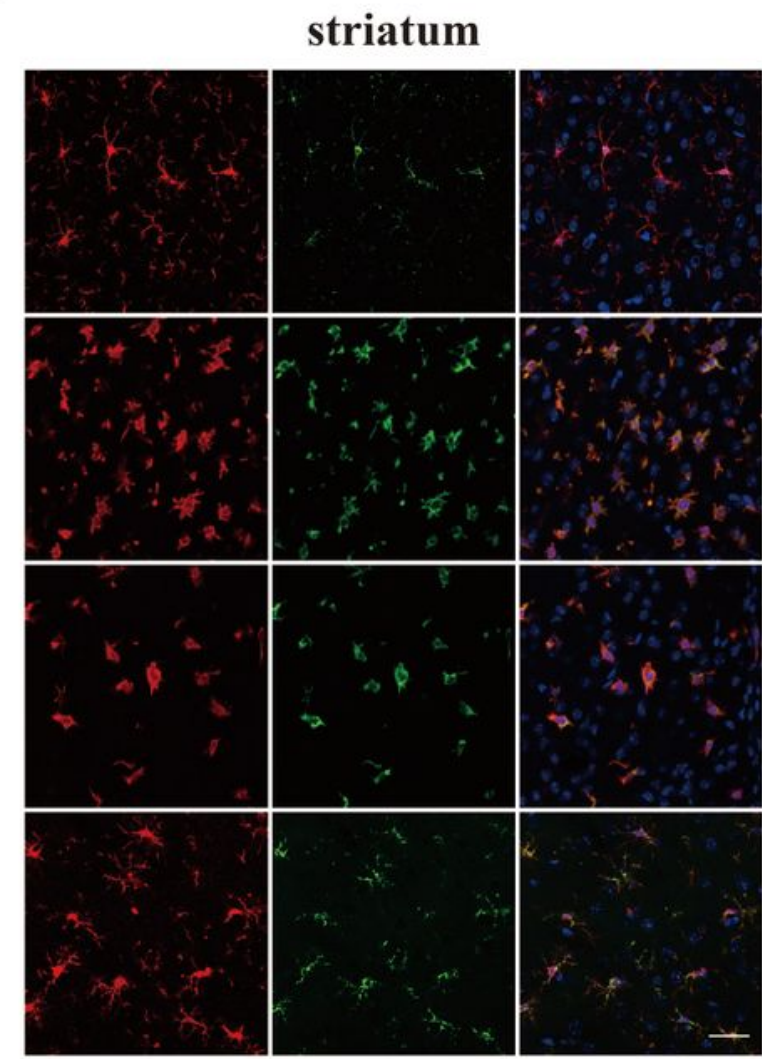

D

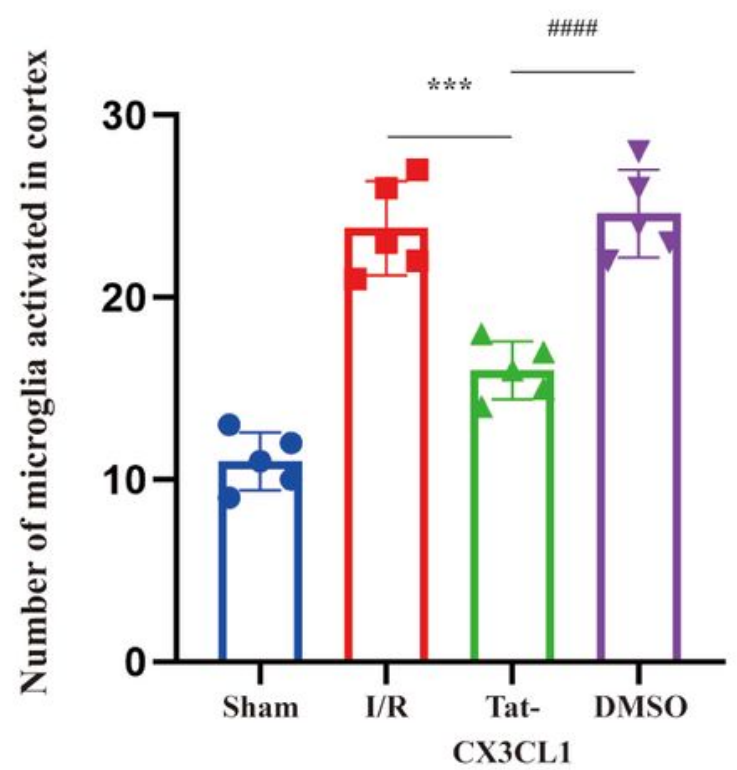

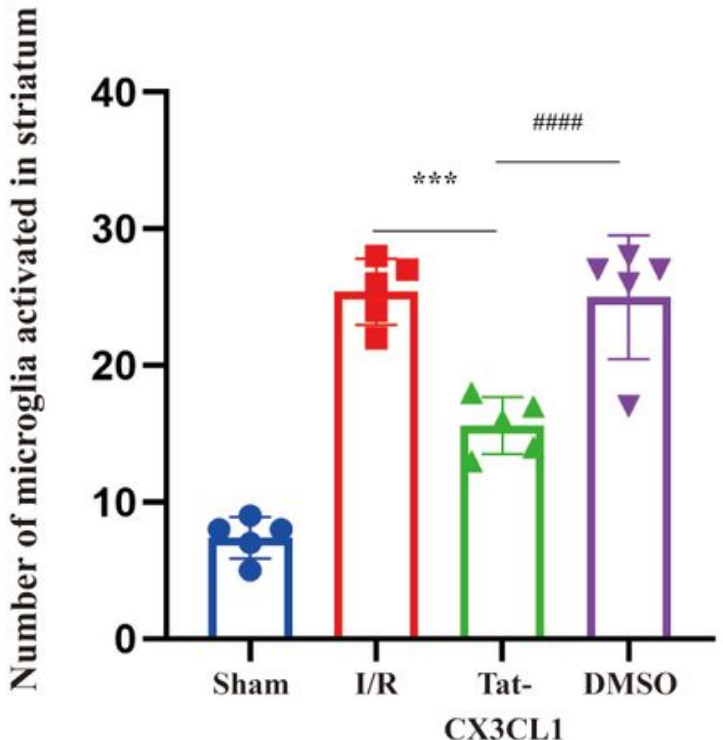

Figure 4

Tat-CX3CL1 (357-395aa) inhibited the polarization of M1-type microglia. (A, B) Representative images of Iba1 (red) and CD68 (green) immunostaining in cortex and striatum at 24h after cerebral ischemia. Scale bar: $50 \mu \mathrm{m}$. Selected areas were observed in cortex and striatum. (C, D) Quantification of the number of 


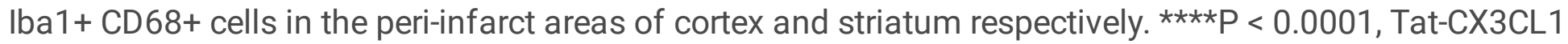
(357-395aa) group versus I/R group; \#\#\#P < 0.001, Tat-CX3CL1 (357-395aa) group versus DMSO group, $\mathrm{n}=5$. Results of data analysis are expressed as means \pm SEM.

A

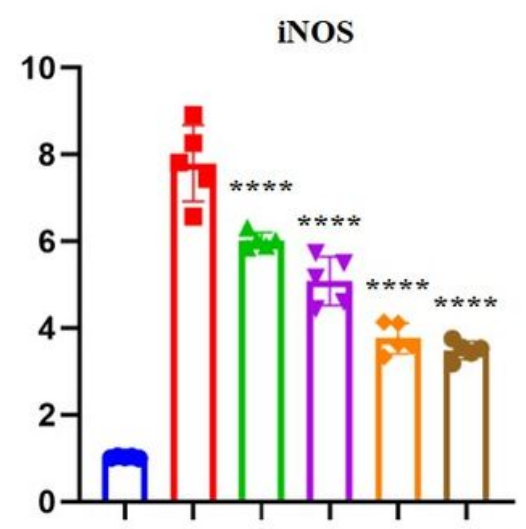

$\begin{array}{lllll}\text { Sham I/R } & 0.25 & 0.5 & 1.0 & 1.5(\mu \mathrm{g} / \mu \mathrm{l})\end{array}$

D

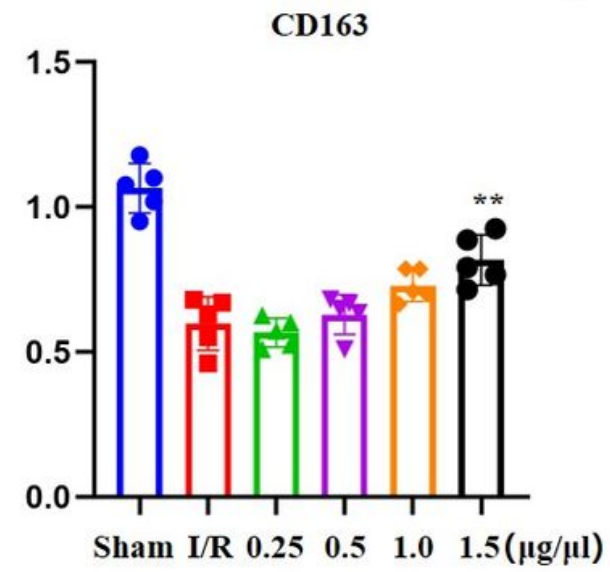

B

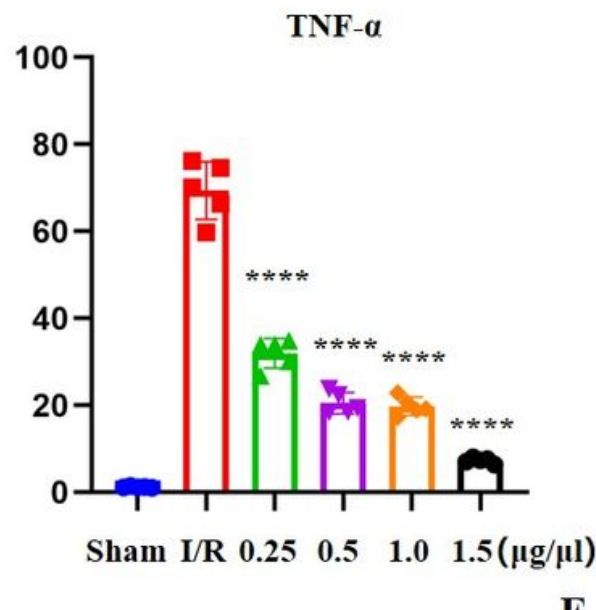

IL-10

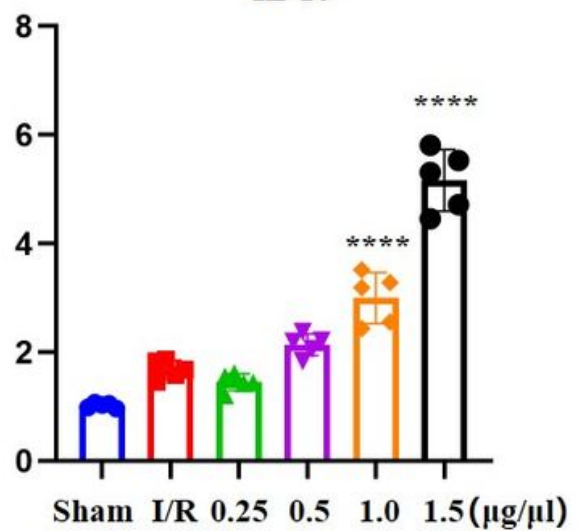

C

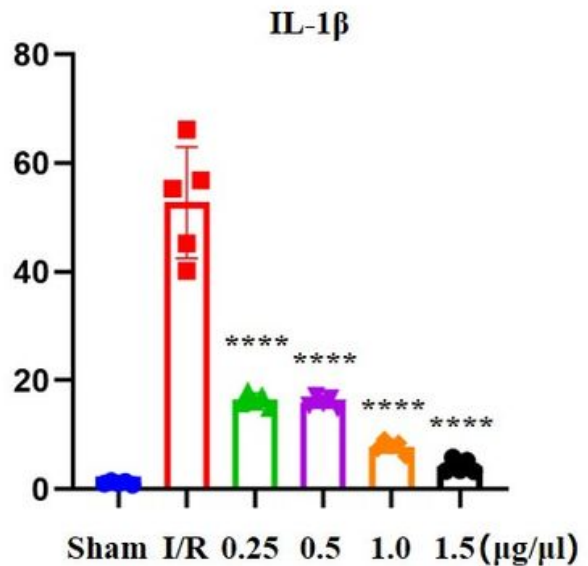

VEGF

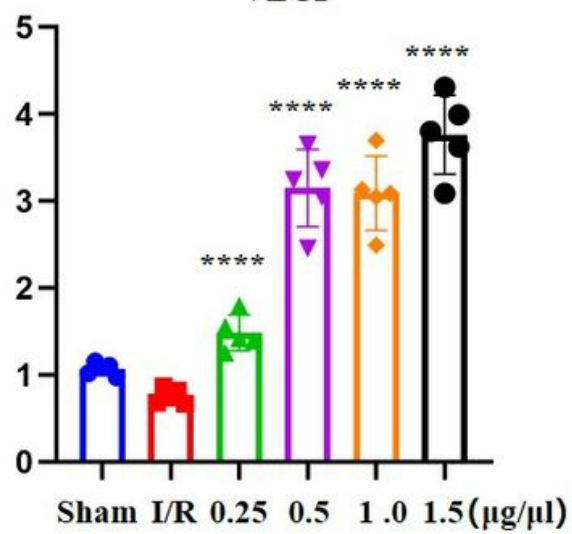

Figure 5

GW280264x, an inhibitor of disintegrin and a metalloproteinase ADAM 17, facilitate the M1 to M2 phenotypic shift at $6 \mathrm{~h}$ after stroke. Different dilution concentrations $(0.25,0.5,1.0,1.5 \mathrm{ug} / \mathrm{ul})$ were selected to ensure the optimal drug concentration. (A-C) Total four-concentration of GW280264x could inhibit the expression of iNOS, TNF-a, IL-1 $\beta$ significantly. (D) Tat-CX3CL1(357-395aa) reduced the escape latency in mice significantly (learning). (E-F) Tat-CX3CL1(357-395aa) extended the time spent in the target quadrant (memory). (D) There was no change in swimming speed in each group. ${ }^{*} \mathrm{P}<0.01$, ${ }^{\star \star \star *} \mathrm{PQ}$ 0.0001, Tat-CX3CL1 (357-395aa) group versus I/R group, $n=5$. 
A

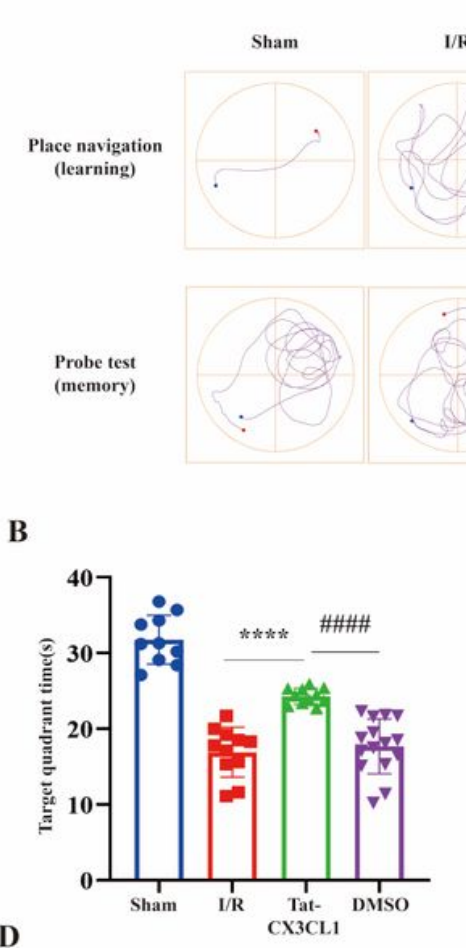

D

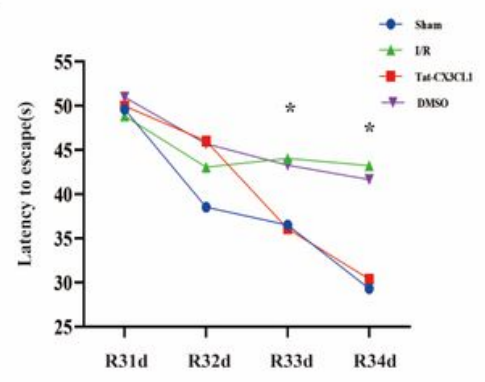

C
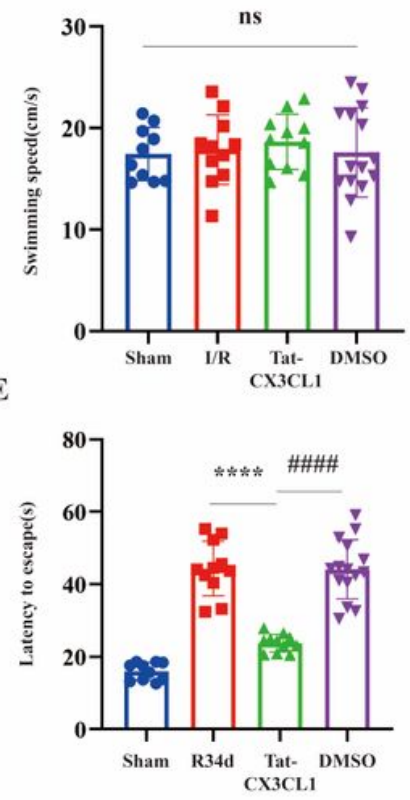

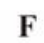

F

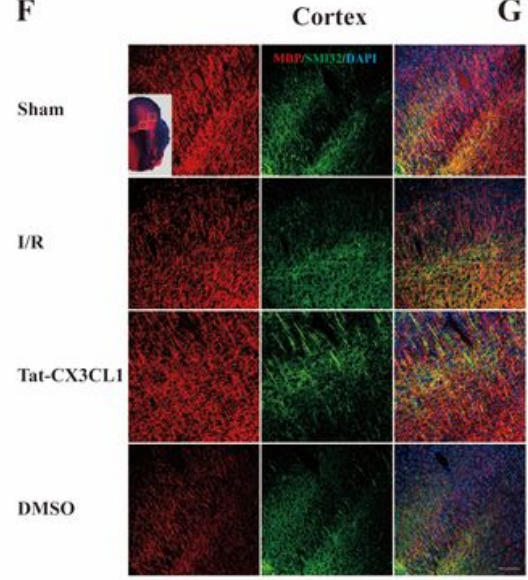

H
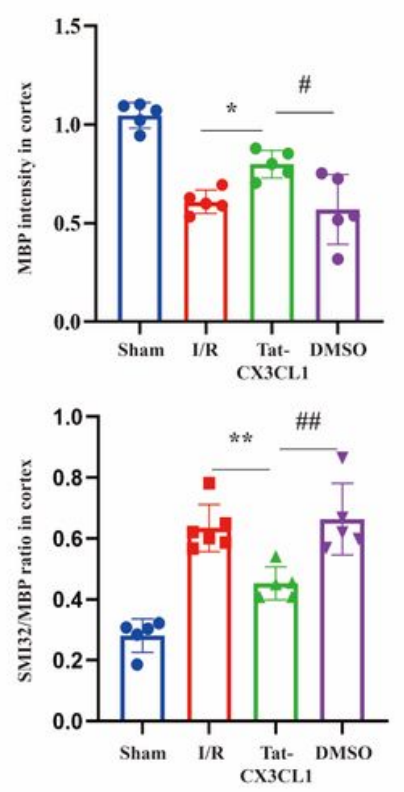

G

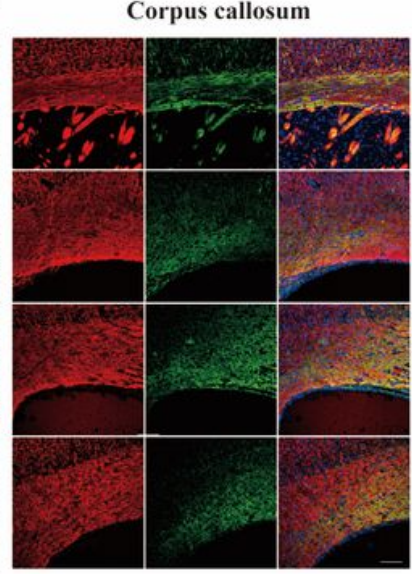

I
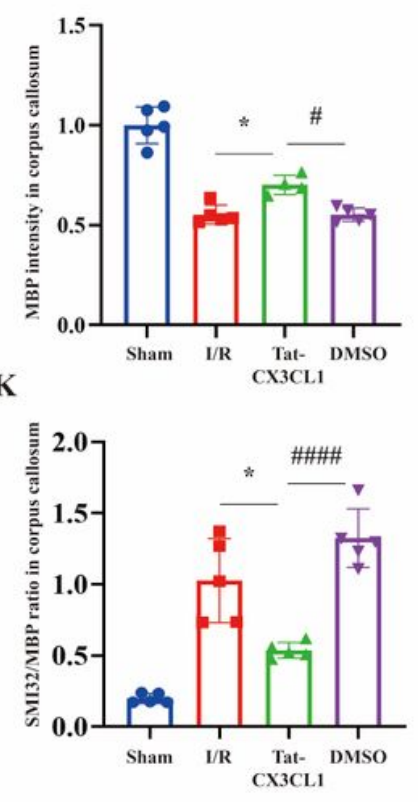

\section{Figure 6}

Tat-CX3CL1 (357-395aa) improved white matter integrity and long-term cognitive functions after MCAO. (A-E) Long-term cognitive functions were evaluated in the Morris water maze. (A) Representative images of the swim paths in each group while the platform was present (learning phase) or removed (memory phase). $n=10-15 /$ group. (B) Tat-CX3CL1 (357-395aa) extended the time spent in the target quadrant (memory). (C) There was no change in swimming speed in each group. (D, E) Tat-CX3CL1 (357-395aa) reduced the escape latency in mice significantly (learning). (F-K) Double immunostaining for MBP and SMI32 at $35 \mathrm{~d}$ after MCAO or sham operation. $n=5 /$ group. Representative images for MBP (red) and SMI32 (green) double immunostaining peri-infarct areas in cortex $(E)$ and CC (F) from sham, l/R group, DMSO group, and Tat-CX3CL1 (357-395aa) group. Selected areas were observed in cortex and striatum. Scale bar: $100 \mu \mathrm{m}$. The fluorescence intensity of MBP in cortex $(\mathrm{H})$ and CC (I). The ratio of SMI32 to MBP immunofluorescence intensity in cortex $(\mathrm{J})$ and $\mathrm{CC}(\mathrm{K})$. Scale bar: $50 \mu \mathrm{m} . \mathrm{n}=5 .{ }^{*} \mathrm{P}<0.05,{ }^{\star *} \mathrm{P}<0.01,{ }^{\star \star * \star *} \mathrm{P}$ $\llbracket 0.0001$, Tat-CX3CL1 (357-395aa) group versus I/R group; \#P<0.05, \#\#P<0.01, \#\#\#\#P<0.0001, TatCX3CL1 (357-395aa) group versus DMSO group. Results of data analysis are expressed as means \pm SEM. 
A

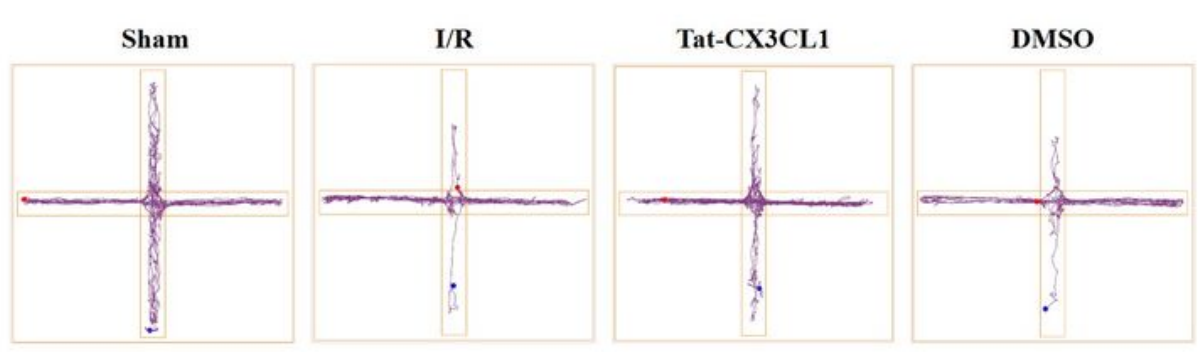

B

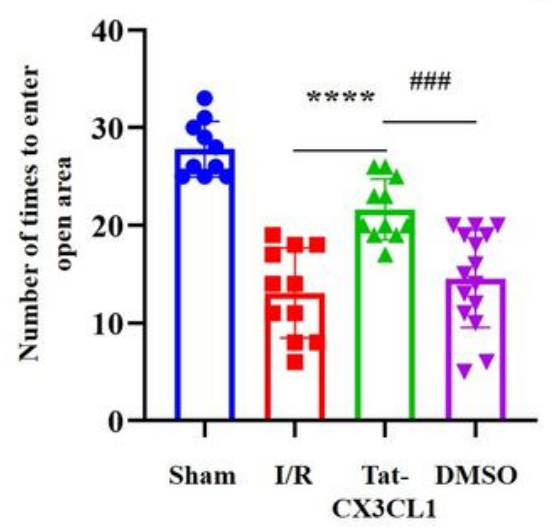

C

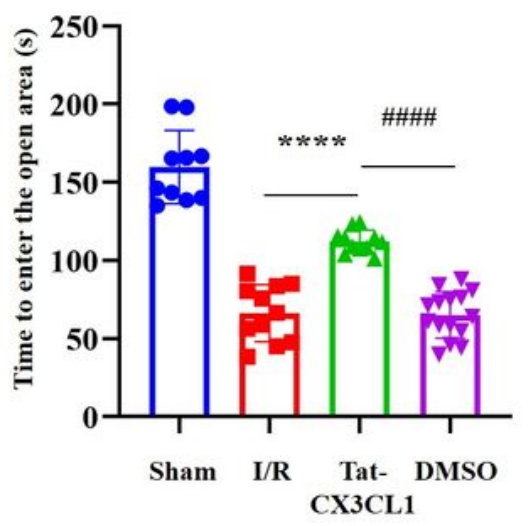

D

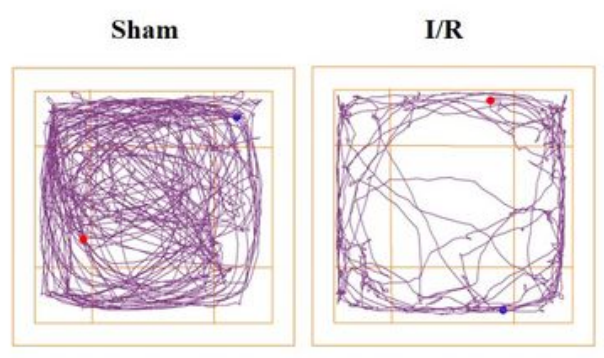

Tat-CX3CL1 DMSO

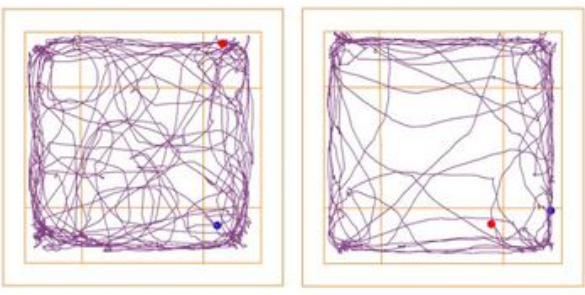

$\mathbf{E}$
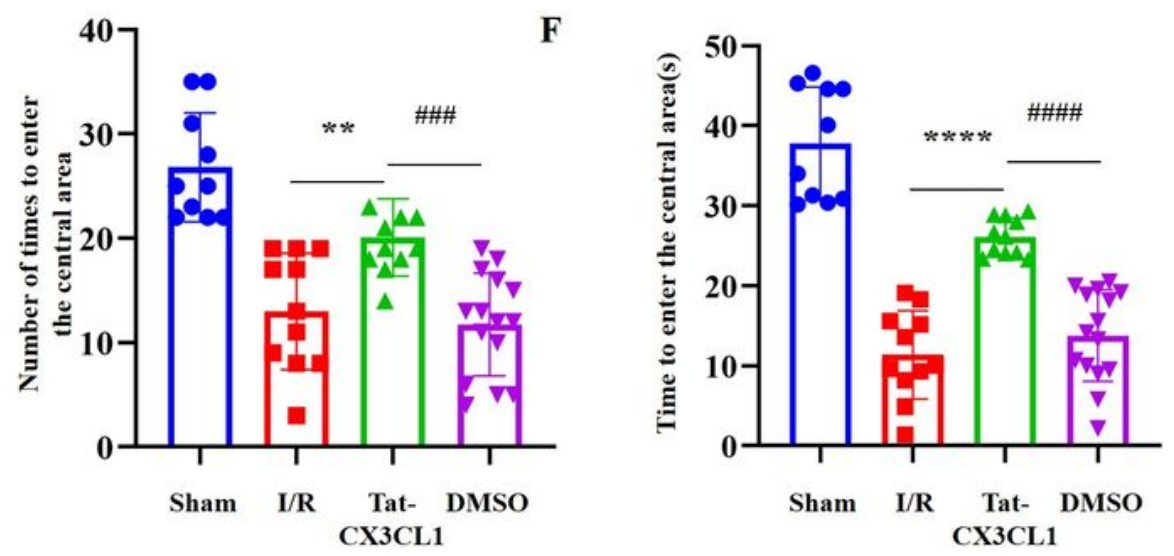

\section{Figure 7}

Tat-CX3CL1 (357-395aa) alleviated the poststroke anxiety and depression in mice. (A) Representative images of movement paths in elevated plus maze in each group. (B, C) Tat-CX3CL1 (357-395aa) increased the number of entering the open arm and the time spent on the open arm. (D) Representative images of movement paths in open field experiment. (E, F) Tat-CX3CL1 (357-395aa) increased the number and time of entering the central region. ${ }^{* *} \mathrm{P}<0.01,{ }^{* * *} \mathrm{P}<0.0001$, Tat-CX3CL1 (357-395aa) group 
versus I/R group; \#\#\#P<0.001, \#\#\#\#P<0.0001, Tat-CX3CL1 (357-395aa) group versus DMSO group, $n=10-15$.

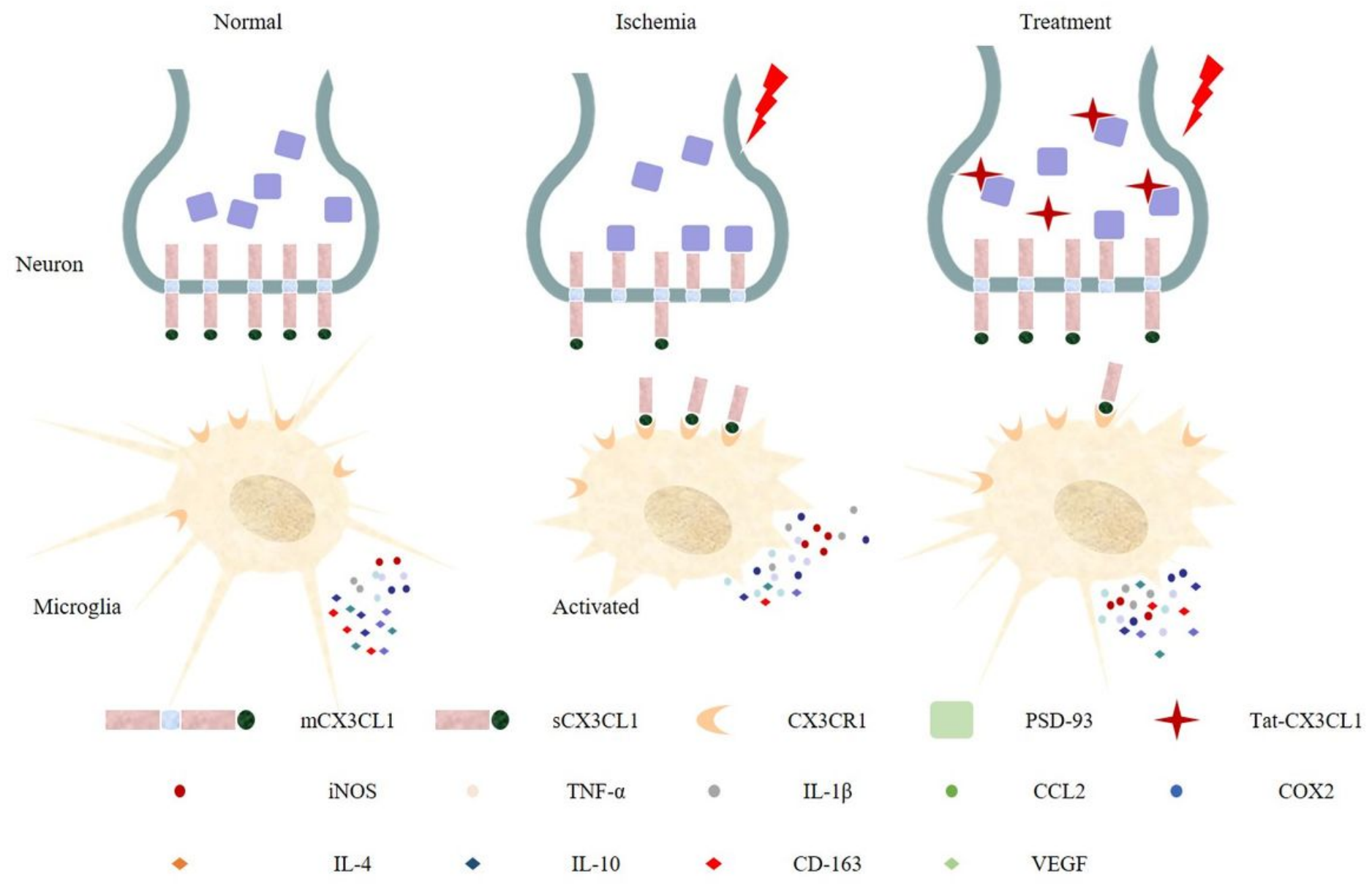

Figure 8

Schematic diagram of Tat-CX3CL1 (357-395aa) performing neuroprotective effect during ischemic cerebral injury. Tat-CX3CL1 (357-395aa) through blocking the combination between PSD-93 and CX3CL1 inhibited the splintering of CX3CL1 into a soluble form and then promoted phenotypic polarization transformation of microglia from M1 type to M2 type.

\section{Supplementary Files}

This is a list of supplementary files associated with this preprint. Click to download.

- Supplemental1.jpg 